Schneider, Thorsten; Dohrmann, Julia

\title{
Religion und Bildungserfolg in Westdeutschland unter besonderer Berücksichtigung von Diasporaeffekten.
}

formal und inhaltlich überarbeitete Version der Originalveröffentlichung in: formally and content revised edition of the original source in:

Kölner Zeitschrift für Soziologie und Sozialpsychologie 67 (2015) 2, S. 293-320

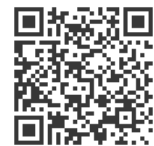

Bitte verwenden Sie in der Quellenangabe folgende URN oder DOI /

Please use the following URN or DOI for reference:

urn:nbn:de:0111-pedocs-157701

10.25656/01:15770

https://nbn-resolving.org/urn:nbn:de:0111-pedocs-157701

https://doi.org/10.25656/01:15770

\section{Nutzungsbedingungen}

Gewährt wird ein nicht exklusives, nicht übertragbares, persönliches und beschränktes Recht auf Nutzung dieses Dokuments. Dieses Dokument ist ausschließlich für den persönlichen, nicht-kommerziellen Gebrauch bestimmt. Die Nutzung stellt keine Übertragung des Eigentumsrechts an diesem Dokument dar und gilt vorbehaltlich der folgenden Einschränkungen Auf sämtlichen Kopien dieses Dokuments müssen alle Urheberrechtshinweise und sonstigen Hinweise auf gesetzlichen Schutz beibehalten werden. Sie dürfen dieses Dokument nicht in irgendeiner Weise abändern, noch dürfen Sie dieses Dokument für öffentliche oder kommerzielle Zwecke vervielfältigen, öffentlich ausstellen, aufführen, vertreiben oder anderweitig nutzen.

Mit der Verwendung dieses Dokuments erkennen Sie die Nutzungsbedingungen an.

\section{Terms of use}

We grant a non-exclusive, non-transferable, individual and limited right to using this document.

This document is solely intended for your personal, non-commercial use. Use of this document does not include any transfer of property rights and it is conditional to the following limitations: All of the copies of this documents must retain all copyright information and other information regarding legal protection. You are not allowed to alter this document in any way, to copy it for public or commercial purposes, to exhibit the document in public, to perform, distribute or otherwise use the document in public.

By using this particular document, you accept the above-stated conditions of use.

\section{Kontakt / Contact:}

\section{peDOCS}

DIPF | Leibniz-Institut für Bildungsforschung und Bildungsinformation Informationszentrum (IZ) Bildung

E-Mail: pedocs@dipf.de

Internet: www.pedocs.de

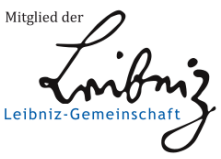




\title{
Religion und Bildungserfolg in Westdeutschland unter besonderer Berücksichtigung von Diasporaeffekten
}

\author{
Thorsten Schneider · Julia Dohrmann
}

Zusammenfassung Die in der quantitativen Bildungssoziologie nahezu verschwundene Kategorie der Religionszugehörigkeit greifen wir auf und fragen, ob und wie diese auch heute noch den Bildungserwerb beeinflusst. Dabei konzentrieren wir uns zunächst auf klassische Erklärungen zu Differenzen in der Lebensführung von Protestanten und Katholiken, auf spezifisches Verhalten in der Diaspora und auf mögliche Selektionseffekte sowie auf muslimische Religionszugehörigkeit als „Bright Boundary“ in Verbindung mit der „Stereotype Threat Theory“. Zur empirischen Überprüfung werden Daten aus dem Sozio-oekonomischen Panel (SOEP) der Jahre 1997 bis 2011 zum Schulbesuch von 13- bis 16-Jährigen in Westdeutschland unter Verwendung von linearen Wahrscheinlichkeitsmodellen mit festen Effekten für Kreise analysiert. Nach Kontrolle sozialstruktureller Merkmale lassen sich keine statistisch signifikanten Differenzen zwischen Katholiken, Protestanten und Muslimen beobachten. Protestanten und Katholiken haben höhere Gymnasialquoten, wenn sie sich in einer regionalen Minderheitensituation befinden. Dies lässt sich am ehesten auf selektive Mobilität der Eltern zurückführen.

Schlüsselwörter Bildungsungleichheiten, Religion, Diaspora, Kontextforschung, Sozio-oekonomisches Panel

\section{T. Schneider}

Institut für Soziologie, Universität Leipzig,

Beethovenstr. 15,

04107 Leipzig, Deutschland

E-Mail:thorsten.schneider@uni-leipzig.de

\section{J. Dohrmann}

Deutsches Institut für Internationale Pädagogische Forschung

(DIPF), Schloßstraße 29,

60486 Frankfurt a. M.,

E-Mail:dohrmann@dipf.de 


\title{
Religious Denomination, and Educational Success in West Germany with a Focus on Diaspora Effects
}

\begin{abstract}
Current research on the relevance of religious affiliation for educational success is scarce in Germany. We address this gap and look at differences among Protestants, Catholics, and Muslims regarding educational success. We focus on West Germany, where both Christian denominations are more or less equal in size overall, but with strong regional variations. According to relevant literature, differences in educational success by religion might be due to specific values and beliefs, or minority status (diaspora), yet parts of the population in a diaspora might be highly selective due to spatial mobility. Muslims might be less successful, as the Islamic religion is seen as a "bright boundary" in the German context, which impedes assimilation. To test these hypotheses, we use data from the German SocioEconomic Panel Study (SOEP) collected between 1997 and 2011. The dependent variable is enrolment in the most prestigious secondary school type ("Gymnasium") at the ages of 13 to 16 . We use linear probability models to estimate school attendance with fixed effects for districts. Altogether, Catholics, Protestants, and Muslims do not differ in educational success if we control for parents' socioeconomic status. In the case of the two Christian denominations, belonging to a regional minority leads to higher success. There are strong hints that this is caused by parents' spatial mobility.
\end{abstract}

Keywords Educational inequality, Religion, Diaspora, Context research, German Socio-economic Panel Study

\section{Einleitung}

In den westdeutschen Diskussionen der 1960er und 1970er Jahre zu „Bildungsnotstand“ (Picht 1964, S. 17) und Bildungschancen hatten das ,katholische Bildungsdefizit" und die Kunstfigur des ,katholischen Arbeitermädchens vom Lande“ (Peisert 1967) feste Plätze (siehe Nellessen-Schumacher 1978; Schmidtchen 1979; Erlinghagen 1965). Müller (1998) zieht in seinem wegweisenden Beitrag „Erwartete und unerwartete Folgen der Bildungsexpansion“ in Zweifel, ob „die Konfessionszugehörigkeit überhaupt nach angemessener Kontrolle der sozialen und regionalen Lage des Elternhauses als eigenständiger Faktor bestand“ (Müller 1998, S. 91), und schenkt diesem Thema konsequenterweise keine Beachtung. In der aktuellen empirischen Bildungsforschung, die von Untersuchungen zu sozialen, geschlechts- und migrationsspezifischen Disparitäten dominiert wird, werden konfessionelle Differenzen ebenfalls kaum thematisiert oder gar empirisch überprüft. Selbst in der Einleitung zum Sonderband der Kölner Zeitschrift für Soziologie und Sozialpsychologie zur soziologischen Bildungsforschung, die einen Abriss der einschlägigen Forschung seit den 1960er Jahren bis heute liefert, werden konfessionelle oder religiös bedingte Disparitäten mit keinem Wort erwähnt (Solga und Becker 2012). Erstaunlich ist dies insofern, als Becker (2007) in einer umfassenden Studie Nachteile in der Bildungsbeteiligung von Katholiken zumindest bis in die 1980er Jahre beobachtet und konfessi- 
onelle Differenzen in Werten und Einstellungen empirisch ermittelt. Er hält fest, dass „[f]ür die Bildungsreproduktion in der Generationenabfolge immer noch die Rolle der Konfessionszugehörigkeit unterschätzt [wurde und wird]“ (Becker 2007, S. 200).

Deshalb untersuchen wir mit aktuellen Daten, ob die Religionszugehörigkeit und regionale Prägung relevante Merkmale für Bildungserfolg sind. Hierbei beziehen wir uns zunächst auf Webers (2006) Ausführungen zur protestantischen Ethik und auf aktuelle Kritik an diesem Ansatz (Becker und Wößmann 2009), um zu verdeutlichen, wie Glaubensinhalte, Vorschriften und seelsorgerische Ratschläge über praktizierte Religion hinaus Einflüsse auf die Lebensführung und die Teilnahme an anderen gesellschaftlichen Teilsystemen haben kann. Selbstverständlich ist zu diskutieren, in wie weit die konkreten Erklärungsmechanismen, die einerseits von Weber, andererseits von Becker und Wößmann herausgearbeitet werden, nach einer längeren Phase der Säkularisierung und der Bildungsexpansion noch zutreffen. Zur fortwährenden Wirkung konfessioneller Zugehörigkeit auf Lebensführung und Einstellungen liegen z. B. aktuelle Studien zur Arbeitsethik und zum politischen Verhalten vor (Basten und Betz 2013; Schaltegger und Torgler 2010).

Wir konzentrierten uns auf den Bildungserfolg von Protestanten und Katholiken in Westdeutschland, weil hier die Mehrheit der Bevölkerung einer der beiden Großkirchen angehört. Obwohl beide in etwa gleich viele Mitglieder haben, bestehen erhebliche regionale Variationen. Dies ermöglicht uns an frühere Forschung zu Diaspora-Effekten anzuschließen, wonach Protestanten und Katholiken dann besonders hohen Bildungserfolg haben, wenn sie in einer regionalen Minderheitensituation leben (Peisert 1967; Schmidtchen 1979). Da der Anteil der Muslime in den letzten Jahrzehnten in Westdeutschland absolut und relativ zugenommen hat, Muslime sich dennoch in einer Minderheitensituation befi prüfen wir, ob auch sie hohe Erfolgsquoten im Bildungssystem aufweisen. Als alternative Erklärung für geringen Bildungserfolg von Muslimen in Deutschland beziehen wir uns auf Albas (2005) Ansatz zu „Bright Boundaries“ und auf die „Stereotype Threat Theory“ (Steele 1997). In einem kleinen Ausblick liefern wir auch Befunde zu konfessionellen Differenzen in der Bildungsbeteiligung in Ostdeutschland, das zu den am stärksten säkularisierten Regionen der Welt gehört.

Für die empirischen Analysen nutzen wir Daten aus dem Sozio-oekonomischen Panel (SOEP), die wir mit Informationen zur regionalen Konfessionsverteilung anreichern. Diese Regionalinformationen haben wir aus Erhebungen des Forsa-Bus generiert. Den Kern der Analyse bilden lineare Wahrscheinlichkeitsmodelle mit festen Effekten für Landkreise und kreisfreie Städte, um das regional variierende Angebot an Schulen sowie die gesamte Infrastruktur, die wirtschaftlichen Bedingungen und sonstigen Besonderheiten statistisch zu kontrollieren.

\section{Protestantische Ethik, Lesegebot und neuere Befunde zu konfessionellen Disparitäten im Bildungserfolg}

Ausgangspunkt unserer Diskussion bildet Max Webers Studie zur Protestantischen Ethik (Weber 2006, S. 23 ff.), der zufolge Protestanten aus Glaubensgründen eine Lebensführung aufweisen, die sich von der der Katholiken unterscheidet und zu höherem Bildungs- und Wirtschaftserfolg führt. In den letzten Jahren haben Becker 
und Wößmann $(2009,2010)$ als Alternativerklärung für beobachtbare Differenzen im Bildungs- und Wirtschaftserfolg zwischen Protestanten und Katholiken im 19. Jahrhundert das Gebot des selbstständigen Lesens der Bibel stark gemacht. Hieraus habe sich eine historisch früher einsetzende Alphabetisierung ergeben (für eine ausführlichere Fassung der Abschn. 2 und 3 mit einer detallierten Darstellung der Argumentation und früherer Befunde vgl. Helbig und Schneider 2014, S. 18, 28-30, 34-43).

Nach Weber (2006, S. 67) bricht Luther mit der damaligen Vorstellung vom Arbeiten als Teil des nicht-religiösen Lebens, um zu überleben oder ein einigermaßen angenehmes Leben zu führen. Der Beruf wird als Berufung, als „eine von Gott gestellte Aufgabe" (Weber 2006, S. 62) gesehen. Noch stärker tritt die Bedeutung religiöser Vorstellungen für berufliches Handeln im Calvinismus hervor. Auf die „pathetische Unmenschlichkeit“ (Weber 2006, S. 88) der Prädestinationslehre schärfte der calvinistische Klerus als seelsorgerischen Ratschlag u. a. rastlose Berufsarbeit ein. Sie allein würde religiöse Zweifel verscheuchen und die Sicherheit des Gnadenstandes geben (Weber 2006, S. 98). Die historische Bedeutung dieser Lebensführung liegt nach Weber in der konsequenten Disziplinierung des täglichen Lebens, mit der eine starke Rationalisierung des Daseins einhergeht (Fitzi 2008, S. 115 f.).

Weber selbst schwankt in seinen Ausführungen, ob sich die Überlegungen zur asketischen Lebensweise auf die stärker lutherisch als calvinistisch geprägten Regionen Deutschlands übertragen lassen (ablehnend z. B. Weber 2006, S. 275). Nach Weber (2006, S. 75) „empfiehlt sich (...), zunächst solche Formen desselben zu betrachten, bei denen ein Zusammenhang der Lebenspraxis mit dem religiösen Ausgangspunkt leichter als beim Luthertum zu ermitteln ist." An einigen Stellen betont er zudem die Rolle des lutherischen Pietismus für die Ausbreitung der asketischen Lebensführung (Weber 2006, S. 123 ff., 281).

Als Alternative zur protestantischen Ethik machen Becker und Wößmann (2009, 2010) das Argument stark, nachdem die Bedeutung des Lesens im Protestantismus Ursache für konfessionelle Differenzen im Bildungs- und Wirtschaftserfolg ist (vgl. Durkheim 1983, S. 176). Luther trat für eine allgemeine Schulpflicht ein, da alle Christen in der Lage sein sollten, das Evangelium zu lesen (Luther 1888, S. 461 f.; Mokrosch 2006, S. 10). Becker und Wößmann (2009) analysieren die Alphabetisierungsraten auf Kreisebene im ausgehenden 19. Jahrhundert in Preußen und stellen fest, dass diese umso höher ausfallen, je mehr Protestanten in einem Kreis leben. Sie zeigen auch, dass die Steuereinnahmen auf Kreisebene mit dem Anteil an Protestanten positiv korrelieren. Wird jedoch die Alphabetisierungsrate berücksichtigt, schwindet die Bedeutung der Konfessionszugehörigkeit. Somit sei keine spezifische Arbeitsethik, sondern eine höhere Bildungsinvestition in protestantischen Familien Ursache für konfessionelle Differenzen im wirtschaftlichen Erfolg.

Sie liefern auch empirische Belege, wonach in Preußen das Schulangebot auf Kreisebene umso dichter und größer war, je höher der Anteil an Protestanten war. Ein höheres Angebot an Schulen sollte zu sinkenden Kosten der Teilnahme führen (Becker und Wößmann 2009, S. 550 ff.). Die Studie enthält aber keine systematische Untersuchung, ob Protestanten und Katholiken innerhalb eines Kreises im gleichen Maße Schulen besuchten, ob es sich also um einen reinen Angebotseffekt handelte.

Seit den Arbeiten Webers sind 100 Jahre vergangen und auch Becker und Wößmanns Analysen beziehen sich auf das 19. Jahrhundert. Seither haben Säkularisie- 
rungsprozesse stattgefunden, die sich z. B. an der Zunahme der Konfessionslosen erkennen lassen. Auch im Bereich der Normen und Werte dürften die Kirchen selbst bei den konfessionell Gebundenen weniger Einfluss haben als vor über $100 \mathrm{Jah}$ ren. Weber (2006) selbst verweist bereits auf Säkularisierungsprozesse und auf die Möglichkeit rationaler, kapitalistisch erfolgreicher Lebensführungen, losgelöst von unmittelbar religiös motivierter asketischer Lebensführung. Schließlich fand ein massiver Ausbau der weiterführenden Bildungsangebote statt. Deshalb stellen wir hier Ergebnisse vor, die sich auf die Nachkriegszeit beziehen.

In der westdeutschen Bildungsforschung wurde vor allem in den 1960er und 1970er Jahren statt vom höheren Bildungserfolg der Protestanten häufig vom katholischen Bildungsdefizit gesprochen (Erlinghagen 1965; Nellessen-Schumacher 1978; Schmidtchen 1979). In einer Auswertung der Volkszählungsdaten aus dem Jahr 1987 stellt Zacharski (1992, S. 401 ff.) fest, dass in Bayern der Anteil der Bevölkerung mit höheren Schul- und Berufsabschlüssen unter den Protestanten höher ist als unter den Katholiken. Beckers (2007) Studie mit Daten aus der westdeutschen Lebensverlaufsstudie zur Bildungsbeteiligung und seine Auswertungen von Allbus-Daten zu Werten und Einstellungen unterstreichen die Differenzen zwischen Katholiken und Protestanten. Bereits Blossfeld (1988) hatte mit der ersten Erhebung der Lebensverlaufsstudie, in der um 1930, 1940 und 1950 geborene Personen retrospektiv befragt wurden, gezeigt, dass Männer und Frauen dann länger im allgemeinbildenden Schulsystem verweilen, wenn das Elternhaus nicht katholisch ist. Dieser Effekt ist in der Arbeit von Blossfeld aber nur bei Frauen statistisch signifikant.

Becker und Wößmann (2009, S. 577 ff.) weisen mit Ende der 1990er Jahre erhobenen Daten des Sozio-oekonomischen Panels (SOEP) nicht nur ein höheres durchschnittliches Bildungsniveau bei Protestanten im Vergleich zu Katholiken nach, sondern auch ein höheres Bruttoerwerbseinkommen. In multivariaten Analysen lassen sich die höheren Einkommen durch das höhere Bildungsniveau erklären. Ein darüber hinausgehender Effekt des Protestantismus lässt sich nicht nachweisen.

Nur wenige Studien haben den Versuch unternommen, die protestantische Ethik mithilfe von Einstellungsvariablen unmittelbar zu messen oder anhand vorliegender Angaben zu Einstellungen nachträglich zu operationalisieren. Letzteres Vorgehen haben Kühnel und Mays (2010) gewählt, die Daten einer Längsschnittkohorte ehemaliger Gymnasiasten in Nordrhein-Westfalen im Hinblick auf die protestantische Ethik auswerten. Die empirischen Befunde verweisen jedoch auf negative Effekte des Arbeitsethos auf den Berufserfolg von Protestanten. Einen positiven Nachweis der protestantischen Arbeitsethik erbringen hingegen Schaltegger und Torgler (2010) mit Daten der European Values Study (EVS) aus den Jahren 1999 und 2000. Hiernach weisen Protestanten in den 16 untersuchten Ländern eine strengere Arbeitsethik als Katholiken auf, wobei diese Differenz bei Protestanten umso stärker ausgeprägt ist, je religiöser sie sich selbst einstufen.

\section{Bildungsbeteiligung in der Diaspora}

Nicht nur im 19. Jahrhundert variierte das Bildungsangebot nach der Konfessionsverteilung, sondern auch für die 1960er Jahre liegen ähnliche Befunde vor. Hoffmann- 
Lange (1984, S. 80) spricht von einer strukturellen Benachteiligung von Katholiken, da ihr Anteil an der Bevölkerung mit abnehmender Gemeindegröße steigt und geschlossene katholische Siedlungsgebiete eher eine traditionelle Wirtschaftsstruktur und folglich auch geringere Bildungsangebote aufweisen (vgl. auch NellessenSchumacher 1978).

Schmidtchen (1979, S. 47 ff.) stellt fest, dass in Bundesländern mit einem hohen Anteil an Katholiken die höheren Schulsysteme schlechter ausgebaut sind als in Bundesländern mit vorwiegend protestantischer Bevölkerung. In weiteren Analysen kommt er zu dem Ergebnis, dass in den Bundesländern mit dem am besten ausgebauten Schulsystem kaum Unterschiede zwischen den Schulbesuchsquoten von Protestanten und Katholiken bestehen. In Bundesländern mit einem mittleren Ausbau des höheren Schulsystems traten Unterschiede zwischen den Konfessionen stärker hervor, wenn ländliche Regionen betrachtet wurden. Dann wiesen Protestanten eine höhere Bildungsbeteiligung auf. In den Bundesländern, die besonders schlecht mit höheren Schulen versorgt waren, ist ein hoher protestantischer Bildungsvorsprung zu beobachten. Schmidtchen (1979, S. 51) schlussfolgert aus seinen Beobachtungen, dass Protestanten vor allem in den Gegenden einen höheren Bildungserfolg aufweisen, in denen sie die Minderheit stellen. Dieser Diaspora-Effekt tritt aber nicht auf, wenn Katholiken in einem Gebiet in der Minderheit sind. Auch Peisert (1967) findet Differenzen in der Bildungsbeteiligung nach konfessioneller Prägung eines Kreises. Er diskutiert aber auch, dass die zu beobachtenden geringen Nachteile für Katholiken möglicherweise auf andere sozialstrukturelle Merkmale zurückzuführen seien und dass es fraglich sei, ob Konfession einen eigenständigen Beitrag leiste (insbesondere S. 89). Zudem basieren seine Analysen auf Aggregatdaten, weshalb hier, wie auch bei Becker und Wößmann (2009), die Gefahr des ökologischen Fehlschlusses besteht.

Wie lassen sich Diaspora-Effekte erklären? Nach Durkheim (1983, S. 167, 171) können Minderheitenstatus und damit eventuell einhergehende Ausgrenzungen ein Zusammenrücken ihrer Mitglieder, stärkere gegenseitige Kontrolle und besonders hohe Anstrengungen bewirken, sich in der Mehrheitsgesellschaft zu behaupten. Das Argument der besonderen Anstrengungen als Ursache für höheren wirtschaftlichen Erfolg wird auch von Weber (2006) in Bezug auf religiöse oder ethnische Minderheiten vorgebracht.

Hier ist auch die Frage zu stellen, wie es überhaupt dazu kommt, dass sich Personen in einer Diaspora befinden. Neben historischen Gründen und Konversion sollten Wanderungsbewegungen eine zentrale Ursache sein. Personen mit einer sehr hohen beruflichen Motivation sollten eher bereit sein, umzuziehen, und je stärker diese Motivation ist, desto weniger sollte die Nähe zum Ort der Kindheit (und die dort vorhandenen sozialen und emotionalen Bindungen) bei Umzugsentscheidungen ins Gewicht fallen. Somit ist anzunehmen, dass mit höherer beruflicher Motivation die Wahrscheinlichkeit steigt, in eine Region zu ziehen, deren konfessionelle Prägung nicht mit der eigenen übereinstimmt. Die hohe berufliche Motivation könnte sich auch auf die Förderung des Nachwuchses und dessen Bildungslaufbahn positiv auswirken (Helbig und Schneider 2014, S. 127).

Aus der Forschung zur Wirkung von Kontexten wissen wir, dass ohne adäquate Berücksichtigung selektiver Mobilität die geschätzten Koeffizienten für Kontexte in der Regel verzerrt sind (Duncan und Raudenbush 2001, S. 358). Deshalb führen z. B. 
Patacchini und Zenou (2007) separate Analysen zum Bildungserfolg von Jugendlichen nach dem Kriterium durch, ob die Eltern schon vor Geburt des Jugendlichen am aktuellen Wohnort lebten oder erst später dorthin gezogen sind. Mit dieser Strategie können sie zeigen, dass Nachbarschaftseffekte stark überschätzt werden, wenn Wanderung nicht beachtet wird. Für die Sesshaften zeigen sich schwache, für die Mobilen stark ausgeprägte Nachbarschaftseffekte. Somit werden originäre Nachbarschaftskontexte in Frage gestellt, denn unbeobachtete Individualmerkmale der Eltern, wie z. B. hohe Bildungsziele für die eigenen Kinder, beeinflussen offenbar die Wahl eines Wohnortes.

\section{Bildungsbeteiligung von Muslimen in Deutschland}

Als Folge von Zuwanderung verzeichnet Deutschland in den letzten Jahrzehnten eine Zunahme an Personen, die sich zum Islam bekennen. Die zuvor diskutierten Diaspora-Effekte, die aus dem Zusammenrücken, der stärkeren Kontrolle und/oder der höheren Motivation, sich zu behaupten, herrühren, sollten auch bei Angehörigen des Islams anzutreffen sein, da sie in Deutschland eine religiöse Minderheit darstellen. Ihre Kinder sollten folglich überdurchschnittlich erfolgreich sein.

Es gibt aber auch Argumente, wonach bei Schülern aus muslimischen Familien mit geringeren Bildungserfolgen zu rechnen ist. ${ }^{1}$ Wir beziehen uns auf den Ansatz von Alba (2005; Foner und Alba 2008; für eine Erweiterung und empirische Prüfung dieses Ansatzes im Arbeitsmarktbereich siehe Connor und Koenig 2013). Ausgangspunkt dieses Ansatzes ist ein Vergleich Westeuropas mit den Vereinigten Staaten. Im Gegensatz zu den Vereinigten Staaten weisen die westeuropäischen Länder einerseits ein hohes Ausmaß an Säkularisierung auf, erkennbar an schwach ausgeprägten religiösen Überzeugungen und Praktiken in der Mehrheitsgesellschaft. Da (nicht-christliche) Zuwanderergruppen in der Regel religiöser sind, weichen sie von der (christlich geprägten) Mehrheitsgesellschaft ab und fallen auf. Andererseits weisen in diesen Ländern die christlichen Kirchen und der Staat, trotz Säkularisierung, spezifische Arrangements auf. Für Deutschland zum Beispiel erkennbar an der Erhebung der Kirchensteuer über die Finanzämter, die Rolle von Kirchen für das Angebot an Kindergärten und Schulen, am Religionsunterricht oder an den christlichen Feiertagen (Alba 2005, S. 32 f.). Dies führt dazu, dass die Zugehörigkeit zum Islam in Deutschland und in Westeuropa (aber nicht in den Vereinigten Staaten) eine „Bright Boundary“, ein klar erkennbares sowie differenzierendes Merkmal ist. „Bright Boundaries“ erschweren den Assimilationsprozess, z. B. die gleichen Teilnahmequoten an weiterführender Bildung oder die gleiche Verteilung von Bildungsabschlüssen wie in der Mehrheitsgesellschaft zu erreichen, weil weder eine schnelle Verschiebung noch eine Verwischung der „Boundaries“ zur Mehrheitsgesellschaft zu erwarten sind.

Wie ist nun die konkrete Erklärung Albas (2005) zu geringeren Bildungserfolgen von muslimischen Schülern? Im westeuropäischen Kontext sei für den Bildungser-

\footnotetext{
${ }^{1} \mathrm{Da}$ in Schulleistungsstudien sowie in administrativen und sozialwissenschaftlichen Befragungen mit Bildungs- und Arbeitsmarktschwerpunkten nur selten Religionszugehörigkeit erhoben wird, liegen zur spezifischen Situation von Muslimen in Deutschland und Westeuropa kaum quantitative Befunde vor.
} 
folg ein „Boundary Crossing“ notwendig. Allgemein geht „Boundary Crossing“ mit einem Verlassen der eigenen Gruppe oder Aufgabe von Zeichen der Zugehörigkeit zu dieser, mit zunehmender Distanz zu den (früheren) Peers, Unsicherheiten über die Akzeptanz durch die neue Gruppe und somit mit sozialen und psychischen Anstrengungen und Belastungen einher (Alba 2005, S. 24). Alba (2005, S. 24 f.) nimmt hier Bezug auf Ogbus (1990; Fordham und Ogbu 1987) „Oppositional Culture Theory“. Schüler, die sich in der Schule „überanpassen“, was als „Acting White“ bezeichnet wird, würden von ihrer eigenen Gruppe wegen mangelnder Loyalität gehänselt. Zudem wäre ihnen noch nicht einmal die Anerkennung durch die „weiße Mehrheit“ in der Schülerschaft und durch die Lehrer sicher.

Albas Rückgriff auf die „Oppositional Culture Theory“ überrascht. Denn Ogbu versucht zu erklären, wieso im Unterschied zu Zuwanderern und ihren (unmittelbaren) Nachkommen z. B. Afro-Amerikaner in den Vereinigten Staaten weit unterdurchschnittlichen Erfolg im Bildungssystem haben. Die Theorie bezieht sich auf Gruppen, die nicht freiwillig migriert sind, die durch Sklavenhandel in ein Land kamen oder kolonialisiert wurden. Dies führt nach Ogbu dazu, dass sich von der Mehrheit abweichende Normen, z. B. Abweichungen von individueller Aufstiegsund Leistungsorientierung, durchsetzen. Freiwillige Migration erfolgt auch nach Ogbu in der Hoffnung auf ein besseres Leben und führt zu hohen Bildungsaspirationen. ${ }^{2}$ Die große Mehrheit der Muslime in Deutschland sind freiwillige Zuwanderer oder deren Kinder und Enkel. Für die Gruppe der Zuwanderer aus der Türkei, die nicht ausschließlich, aber überwiegend Muslime sind, liegen gut dokumentierte Befunde zu ihren hohen Bildungsaspirationen vor (Relikowski et al. 2012).

Deshalb erscheint der von Alba vorgeschlagene Weg über die „Oppositional Culture Theory" nicht tragfähig. Der Ausgangspunkt, dass es sich bei Angehörigen des Islams um eine klar (selbst- und fremd-)identifizierbare Gruppe handelt, könnte dennoch hilfreich sein. Die wenig religiöse Mehrheitsgesellschaft und die gleichzeitig sichtbare Verankerung der christlichen Traditionen auch im Schulalltag (Religionsunterricht, Gottesdienste zum Schuljahresanfang und -ende, Schulferien an christlichen Feiertagen etc.) könnten zu einem Unwohlsein, zu Gefühlen des nicht vollständigen Willkommen-Seins und des Andersseins in der Schule führen. Dies muss sich nicht unmittelbar auf weitreichende Bildungsziele auswirken, sondern könnte die Leistungsentwicklung hemmen. Nach der "Stereotype Threat Theory“ schneiden benachteiligte Gruppen in Testsituationen dann schlechter ab, wenn ihnen die Gruppenzugehörigkeit und ihre vermeintliche „Inferiorität“ oder die besondere Wichtigkeit der Testsituation verdeutlicht wird. Es wird angenommen, dass die Aktivierung der Zugehörigkeit zu solch einer Gruppe die Aufmerksamkeitskonzentration bei der Bearbeitung eines Tests senkt und deshalb zu geringeren Ergebnissen führt (Steele 1997). Ob der „Stereotype Threat“ auch langfristige Konsequenzen für Interessen- und Leistungsentwicklung hat, wurde nur selten überprüft, in den wenigen

\footnotetext{
${ }^{2}$ Darüber hinaus ist die Gültigkeit der „Oppositional Culture Theory“ stark umstritten. Ainsworth-Darnell und Douglas (1998) haben mit US-amerikanischen Daten versucht, die Theorie zu prüfen. Sie fanden weder Hinweise dafür, dass afro-amerikanische Schüler niedrigere Bildungs- und Berufsziele haben, noch dass leistungsstarke Schüler aus dieser Gruppe unpopulär sind, wie nach dem Argument des „Acting White"zu erwarten wäre.
} 
vorliegenden Studien aber durch empirische Befunde gestützt (Kiefer und Sekaquaptewa 2007; Woodcock et al. 2012).

\section{Forschungsfragen und Hypothesen}

Vor dem Hintergrund der vorgestellten Theorien und Befunde werden zwei Forschungsfragen und zwei Hypothesen aufgestellt.

Die erste Forschungsfrage lautet: Haben Protestanten einen größeren Bildungserfolg als Katholiken?

Nach der Protestantischen Ethik kommen als Mechanismen eine höhere Disziplin und die Bereitschaft für längerfristige Investitionen in Frage, die sich sowohl auf den Erwerb (schulischer) Kompetenzen als auch auf Bildungsentscheidungen auswirken sollten. Nach dem Argument des Lesegebots wären hingegen Differenzen in den Leseleistungen zu erwarten, die wiederum den Bildungserfolg beflügeln.

Die zweite Forschungsfrage lautet: Haben Kinder aus muslimischen Familien geringe Bildungserfolge als Kinder aus christlichen Familien?

Sie könnten geringere Fortschritte in der Leistungsentwicklung aufweisen, denn institutionell verankerte Praktiken, die den beiden christlichen Großkirchen im Schulalltag eine Sonderstellung einräumen, verdeutlichen, dass die muslimische Religionszugehörigkeit eine „Bright Boundary“ ist, entlang derer sich Stereotype etablieren könnten.

Diaspora-Effekte.

H1: Nach Argumenten von Durkheim erwarten wir, dass Mitglieder einer Religion erfolgreicher sind, wenn sie sich in einer Minderheitensituation befinden, weil entweder die Gemeinschaft stärker zusammenrückt und somit auch den Nachwuchs stärker kontrolliert oder weil die Angehörigen der Minderheitenreligion ein stärkeres Bedürfnis haben, sich in der Mehrheitsgesellschaft zu behaupten. Diese Mechanismen sollten auf Katholiken, Protestanten und Muslime gleichermaßen zutreffen.

H2: Alternativ könnte ein beobachtbarer Diaspora-Effekt das Ergebnis selektiver Wanderungsbewegungen sein und auf hohe berufliche Motivation und Bildungsaspirationen der Eltern verweisen. Deshalb sollte sich der Diaspora-Effekt nicht in der Gruppe der Ortsansässigen, sondern nur in der Gruppe der räumlich Mobilen zeigen.

\section{Daten, Operationalisierungen und Methoden}

\subsection{Nutzung der SOEP-Daten und Variablenkonstruktion}

Zur Analyse der Bedeutung von Religion für Bildungserfolg verwenden wir im Schwerpunkt Daten aus dem SOEP, Version 29 (SOEP 2012; Wagner et al. 2008). Wir beziehen uns auf Angaben, die in den Jahren 1997, 2003, 2007 und 2011 erhoben wurden, weil in diesen Jahren Fragen zur Religionszugehörigkeit gestellt wurden. 
Die Angaben aus den vier Erhebungsjahren werden gepoolt, wobei jeder Jugendliche nur einmal beobachtet wird, Mütter (und Väter) zum Teil aber mehrfach (siehe hierzu Abschn. 6.2; zu einer früheren Fassung der ersten vier Absätze in Abschn. 6.1 mit einer deutlich begrenzteren Datenlage siehe Helbig und Schneider 2014, S. 58 f.).

Als Indikator für den Bildungserfolg verwenden wir den Gymnasialbesuch von Jugendlichen, die im jeweiligen Erhebungsjahr das 13., 14., 15. oder 16. Lebensjahr vollenden. Alle Jugendlichen, die eine andere Schulart in der Sekundarstufe besuchen, gehören zur Referenzkategorie. Die abhängige Variable ist somit binär kodiert. $\mathrm{Zu}$ den Jugendlichen werden die Angaben ihrer Mutter und ihres Vaters oder die des aktuellen Partners der Mutter zugespielt.

Weil deutlich mehr Angaben zu Müttern als zu Vätern vorliegen und weil wir eine übersichtliche und zugleich sparsame Modellierung der zu prüfenden Zusammenhänge anstreben, beziehen wir uns primär auf die Angabe zur Religionszugehörigkeit der Mutter. Wir differenzieren nach katholisch (Referenzkategorie), protestantisch, muslimisch, sonstige Religionszugehörigkeit und konfessionslos (kein Mitglied einer Religionsgemeinschaft). Angaben zur muslimischen Religionszugehörigkeit stehen für das Jahr 1997 nicht zu Verfügung, weshalb alle Frauen, für die 1997 die Angabe „sonstige Religionszugehörigkeit“ und für 2003 die Angabe „muslimisch“ vorliegt, die 1997er Angabe auf muslimisch rekodiert wird. In Einzelfällen, wenn z. B. ein Ausstieg aus dem Panel bis zum Jahr 2003 erfolgt ist, werden muslimische Familien somit untererfasst. Zusätzlich generieren wir zwei Dummy-Variablen, mit denen erfasst wird, ob beide Elternteile katholisch oder evangelisch sind. Die Angaben auf die Frage nach der Häufigkeit von „Kirchgang, Besuch religiöser Veranstaltungen" wird in den Jahren 1997, 2007 und 2011 mit einer 4-stufigen Antwortskala (,jede Woche“, ,jeden Monat“, „seltener“, „nie“) und 2003 mit einer fünfstufigen erfasst („täglich“, „mindestens 1-mal pro Woche“, „mindestens 1-mal pro Monat“, „seltener", „nie“). Die beiden Antwortkategorien, die sich in der 5er-Skala auf die häufigsten Aktivitäten beziehen, werden zusammengefasst und folgende Kodierung für die Angaben aus allen vier Erhebungsjahren vergeben: $0=$ nie, $1=$ seltener, $2=$ jeden Monat, $3=$ jede Woche.

Um auszuschließen, dass etwaige Differenzen im Schulerfolg der Kinder nach Religionszugehörigkeit lediglich auf Unterschieden in der sozio-oekonomischen Herkunft und den kulturellen Aktivitäten der Eltern beruhen, kontrollieren wir für in der Bildungsforschung gängige Strukturmerkmale und einige wenige Prozessmerkmale (Jungbauer-Gans et al. 2012). Der Bildungsabschluss der Eltern wird anhand der Anzahl der idealtypischen Bildungsjahre mit Ausprägungen von 7 (ohne jeden Abschluss) bis 18 (Universitätsabschluss) und die berufliche Position anhand des International Socio-Economic Index (ISEI; Ganzeboom und Treiman 2003) gemessen, wobei im Falle von zwei gültigen Elternangaben der jeweils höhere Wert verwendet wird. Zudem kontrollieren wir die Häufigkeit des „Besuch[s] von kulturellen Veranstaltungen, z. B. Konzerten, Theater, Vorträgen" der Mutter, die ebenfalls in den Jahren 1997, 2003, 2007 und 2011 unter Verwendung der bereits am Beispiel des Kirchgangs beschriebenen Antwortskalen erhoben wurden. Die Antworten werden in der weiter oben vorgestellten 4-stufigen Skala abgelegt und als quasi metrische Angabe verwendet. Zudem kontrollieren wir, ob der Vater im Haushalt lebt und ob er an der Befragung teilgenommen hat. Hierzu bilden wir eine Dummy-Variable „kein 
Vater im Haushalt (HH)“ und „Vater im HH, aber keine Teilnahme (TN)“ an der SOEP-Befragung. Der Migrationshintergrund wird über das Geburtsland der Eltern operationalisiert: Eine Dummy-Variable bezieht sich auf Fälle, in denen genau ein Elternteil im Ausland geboren wurde, eine weitere auf Fälle, in denen beide Elternteile im Ausland geboren wurden. Schließlich kontrollieren wir für das Geschlecht des Kindes (Junge $=1$, Mädchen $=0$ ), die Anzahl der leiblichen Geschwister mütterlicherseits und für das Erhebungsjahr mithilfe von Dummy-Variablen.

Für Analysen zur Bedeutung der Diaspora greifen wir auf zwei weitere Informationen zurück, nämlich zum Leben am Ort der Kindheit und zum Geburtsort. Im SOEP werden Personen i. d. R in einer der ersten Erhebungen gefragt, ob sie immer noch oder wieder am Ort der Kindheit wohnen oder an einem anderen Ort. Bei dieser Frage gibt es auffällig viele fehlende Werte in dem 1984 gestarteten, auf die „Gastarbeiterpopulation" ausgerichteten Subsample B und in dem Mitte der 1990er Jahre gestarteten Subsample D zu Neuzuwanderern. Wir verwenden hier die Angabe der Mutter. Zwischen dieser Angabe und dem beobachteten Schulbesuch ihres Kindes können mehrere Jahre liegen, sodass es Unschärfen geben kann. Im Jahr 2012 wurden die Befragten gebeten, ihren Geburtsort anzugeben. Für in Deutschland geborene Personen wurde diese Angabe mit Kreiskennziffern vercodet (nach heutigem Gebietsstand). Für Personen, die im Jahr 2012 nicht (mehr) an der SOEP-Studie teilnahmen, liegen keine Angaben vor, und für Personen, bei denen der Geburtsort nicht eindeutig zugeordnet werden konnte, liegen mehrere mögliche Kreiskennziffern vor. Beide Fallkategorien müssen aus der Analyse ausgeschlossen werden. Über Abgleich des aktuellen Wohnkreises der Mutter mit dem Kreis, in dem sie geboren wurde, kann zwischen Müttern differenziert werden, die sesshaft und mobil sind. Bei den Mobilen unterscheiden wir noch einmal zusätzlich zwischen denen, bei denen im Geburtskreis mehr als $40 \%$ der (aktuellen) Bevölkerung zur eigenen Konfession gehören, im aktuellen Wohnkreis aber weniger als 30\% („Umzug in Diaspora“), und denen, bei denen im Geburtskreis weniger als $30 \%$ der (aktuellen) Bevölkerung zur eigenen Konfession gehören, im aktuellen Wohnkreis aber mehr als $40 \%$ (,Auszug aus der Diaspora"), und allen anderen Mobilen. Zusätzlich verwenden wir diese Informationen, um Distanzen zwischen dem Kreis, in dem eine Mutter geboren wurde, und dem Kreis, in dem sie heute lebt, zu erfassen.

Für Westdeutschland ergibt sich aus den SOEP-Daten zunächst ein Stichprobenumfang von $n=4051$ Jugendlichen. In fast $4 \%$ der Fälle liegen keine Angaben zur Mutter vor, weil sie entweder nicht im Haushalt lebt oder bereits verstorben ist $(n=72)$ oder an der Befragung nicht teilgenommen hat $(n=85)$. Weitere Ausfälle entstehen, weil keine gültige Angabe zum Schulbesuch vorliegt $(n=112)$ oder weil Kinder noch zur Grundschule gehen und somit noch keine Sekundarschule besuchen $(n=18)$. Einige wenige ungültige Angaben liegen für die Religionszugehörigkeit, den Kirchgang und die hochkulturellen Aktivitäten der Mutter sowie für den höchsten Bildungsabschluss vor. Nach fallweisem Ausschluss beträgt der Stichprobenumfang $n=3622$.

Im Fall des höchsten ISEI der Eltern sind häufiger fehlende Werte zu beobachten. Diese werden durch eine regressionsbasierte Einmal-Imputation unter Nutzung des verfügbaren Nettohaushaltsäquivalenzeinkommens, der Bildung der Eltern, ihrem 
Migrationshintergrund und dem Merkmal „Alleinerziehend“ sowie unter Addition eines Zufallsterms aus der modellbasierten Residuenverteilung ersetzt.

Da mit den Mikro-Daten des SOEP Diaspora-Effekte überprüft werden sollen, müssen regionale Informationen zugespiegelt werden. Hierfür aggregieren wir Individualdaten aus dem Forsa-Bus der Jahre 1997-2007 (FORSA 2008) und bestimmen so den Anteil von Katholiken auf Kreisebene. Die sehr hohen Fallzahlen des ForsaBus erlauben eine zuverlässlige Schätzung des Anteils an Katholiken auf Kreisebene (ausführliche Informationen hierzu bei Helbig und Schneider 2014, S. 60 f.).

\subsection{Statistische Methode: Fixed-Effects-Modelle}

Um festzustellen, ob Religionszugehörigkeit Einfluss auf den Bildungserfolg hat, wenden wir die Strategie der Drittvariablenkontrolle an. Wir berücksichtigen in der Modellierung z. B. das Bildungsniveau der Eltern, da dies mit den beiden zentralen Merkmalen korrelieren könnte und im Falle einer Nichtberücksichtigung verzerrte Effekte geschätzt werden könnten. Da auch regionale Merkmale mit Individualmerkmalen korrelieren könnten, kann auch deren Nichtberücksichtigung zu verzerrten Schätzungen führen. Zudem zielt die Untersuchung darauf zu prüfen, ob Katholiken, Protestanten und Muslime sich unterscheiden, wenn sie das gleiche Angebot vor Ort haben. Deshalb schätzen wir Lineare Wahrscheinlichkeitsmodelle (LPM) mit festen Effekten für Kreise. Implizit nehmen wir an, dass Kreisstrukturen (z. B. Angebote an Bildungseinrichtungen, Verkehrsverbindungen) eine für Bildungsentscheidungen relevante räumliche Einheit darstellen und rechnen alle zeitkonstanten Kreismerkmale raus (für eine ausführlichere Fassung dieses Abschnitts siehe Helbig und Schneider 2014, S. 61 f.).

Zusätzlich werden die Standardfehler nach dem Sandwich-Huber-Verfahren korrigiert, weil zu den 3622 Jugendlichen 2511 Mütter gehören, einige Mütter also mehrfach beobachtet werden (Wooldridge 2000, S. 248-255).

\section{Ergebnisse}

Zunächst werden einige ausgewählte bivariate Befunde präsentiert, dann sehr ausführlich die multivariaten Ergebnisse, und zwar in der Abfolge: grundlegender Aufbau der Modellstruktur, Relevanz der Religionszugehörigkeit und der Diaspora. Abschließend gibt es einen kleinen Exkurs zu Ostdeutschland, da es zu den am stärksten säkularisierten Regionen der Welt gehört und Religionsangehörige sich dort in einer Diaspora befinden.

In Tab. 1 ist der Anteil der Gymnasiasten in Westdeutschland in Abhängigkeit von der Religionszugehörigkeit der Mutter dargestellt. Im Durchschnitt besuchen 37\% der Jugendlichen mit einer katholischen, 39\% mit einer evangelischen und 12\% mit einer muslimischen Mutter das Gymnasium. Gehört die Mutter einer sonstigen Religionsgemeinschaft an, beträgt die Wahrscheinlichkeit des Gymnasialbesuchs $26 \%$, im Fall einer konfessionslosen Mutter 37\%.

Bleiben die Differenzen bestehen, wenn im Rahmen des linearen Wahrscheinlichkeitsmodells alle zeitkonstanten Merkmale auf Kreisebene herausgerechnet werden? 
Tab. 1 Anteil an Gymnasiasten nach Religionszugehörigkeit der Mutter in Westdeutschland. (Quellen: SOEP, v29; eigene Berechnungen; Gym Gymnasialquote; $n$ Fallzahlen)

\begin{tabular}{lll}
\hline Religion & Gym (\%) & $n$ \\
\hline Katholisch & 37 & 1400 \\
Evangelisch & 39 & 1349 \\
Muslimisch & 12 & 259 \\
Sonstige & 26 & 189 \\
Konfessionslos & 37 & 425 \\
\hline Insgesamt & 36 & 3622 \\
\hline
\end{tabular}

Tabelle 2 weist in der zweiten Spalte die Bruttoeffekte aus, d. h. es werden nur Variablen, die zu einem Merkmal gehören, berücksichtigt. Somit enthält die zweite Spalte in Tab. 2 die Ergebnisse aus 9 getrennten Modellschätzungen. Während nach Tab. 1 die Gymnasialquoten von Protestanten leicht höher ausfallen als die von Katholiken, haben sie nach dem linearen Wahrscheinlichkeitsmodell mit festen Effekten für Kreise eine um 1,3\%-Punkte geringere Wahrscheinlichkeit $(\beta=-0,013)$, diese Schulart zu besuchen. Diese Differenz ist statistisch nicht signifikant. Deutlicher treten die Unterschiede in den Quoten ohne und mit Berücksichtigung der Kreise bei einer Beschränkung auf Bayern hervor (Schätzungen nicht in Tabellenform ausgewiesen). In Bayern fällt die Gymnasialquote für Protestanten gegenüber Katholiken um 7,3 \%-Punkte höher aus (wenn auch nicht signifikant). In den Modellen mit festen Kreiseffekten schrumpft die Differenz auf 1,8 \%-Punkte. Protestanten und Katholiken verteilen sich offenbar unterschiedlich über die bayerischen Kreise. Ihre Bildungsbeteiligungsquoten unterscheiden sich aber nicht, wenn sie im selben Kreis leben.

Wenn Kovariaten zur sozialstrukturellen Lage der Familie berücksichtigt werden, ist der Schätzkoeffizient für evangelische im Vergleich zu katholischen Müttern weiterhin nicht signifikant (siehe Modell 1, Tab. 2). Der Effekt für muslimische Mütter reduziert sich drastisch von $-0,288$ auf $-0,056$. Der neue Koeffizient ist nicht mehr statistisch signifikant. Die zunächst beobachtbare Differenz zwischen Muslimen und Katholiken ist somit vollständig auf sozialstrukturelle Merkmale zurückzuführen. ${ }^{3}$

Nach diesen Befunden können die ersten beiden Forschungsfragen, ob Jugendliche aus protestantischen und katholischen Familien einerseits sowie aus muslimischen und christlichen Familien andererseits differierende Beteiligungsquoten haben, verneint werden, sofern für sozialstrukturelle Merkmale kontrolliert wird.

Im Folgenden möchten wir die Stabilität der Befunde mit zwei weiteren Modellierungsstrategien überprüfen. Im Fall von Protestanten und Katholiken könnten Differenzen dann zum Tragen kommen, wenn protestantische Werte und Einstellungen besonders stark verinnerlicht sind. Die Wahrscheinlichkeit, dass dies der Fall ist, sollte in religiös homogamen Elternhäusern größer sein. Deshalb erfassen wir mit jeweils zwei Dummy-Variablen, ob beide Eltern protestantisch oder katholisch sind. (Da muslimische Eltern sehr hohe Raten an religiöser Homogamie aufweisen, ist eine weitere Dummy-Variable für diese Gruppe nicht möglich.) Im Falle religiöser Homogamie werden sowohl für katholische als auch für evangelische Eltern positive und signifikante Effekte ausgewiesen (siehe Modell 2, Tab. 3). Entgegen der Vermutung ist der Effekt für evangelische Elternpaare aber kleiner als der für katholische Paare,

\footnotetext{
${ }^{3}$ Die Effekte, die für die Kontrollvariablen ausgewiesen werden, entsprechen dem Stand der Forschung.
} 
Tab. 2 Gymnasialbesuch in Westdeutschland - Lineares Wahrscheinlichkeitsmodell mit fixen Effekten für Kreise. (Quellen: SOEP, v29; eigene Berechnungen)

\begin{tabular}{|c|c|c|}
\hline & Bruttoeffekte & Modell 1 \\
\hline \multicolumn{3}{|c|}{ Religion der Mutter (Ref.: katholisch) } \\
\hline \multirow[t]{2}{*}{ Evangelisch } & $-0,013$ & $-0,030$ \\
\hline & $(0,025)$ & $(0,021)$ \\
\hline \multirow[t]{2}{*}{ Muslimisch } & $-0,288 * *$ & $-0,056$ \\
\hline & $(0,036)$ & $(0,037)$ \\
\hline \multirow[t]{2}{*}{ Sonstige } & $-0,158 * *$ & $-0,079^{+}$ \\
\hline & $(0,043)$ & $(0,042)$ \\
\hline \multirow[t]{2}{*}{ Konfessionslos } & $-0,046$ & $-0,072 *$ \\
\hline & $(0,032)$ & $(0,029)$ \\
\hline \multirow[t]{2}{*}{ Häufigkeit Hochkultur (linear) } & $0,209 * *$ & $0,077 * *$ \\
\hline & $(0,014)$ & $(0,014)$ \\
\hline \multirow[t]{2}{*}{ Anzahl Bildungsjahre (Eltern) ${ }^{\mathrm{a}}$} & $0,078 * *$ & $0,054 * *$ \\
\hline & $(0,003)$ & $(0,004)$ \\
\hline \multirow[t]{2}{*}{ Höchster ISEI (Eltern) ${ }^{\mathrm{a}}$} & $0,011 * *$ & $0,004 * *$ \\
\hline & $(0,000)$ & $(0,001)$ \\
\hline \multicolumn{3}{|l|}{ Status Partner der Mutter/Vater } \\
\hline \multicolumn{3}{|l|}{ (Ref.: im HH und Interview) } \\
\hline \multirow[t]{2}{*}{ nicht im $\mathrm{HH}$} & $-0,112 * *$ & 0,030 \\
\hline & $(0,027)$ & $(0,025)$ \\
\hline \multirow[t]{2}{*}{ im $\mathrm{HH}$, aber kein Interview } & 0,028 & $0,063^{+}$ \\
\hline & $(0,042)$ & $(0,037)$ \\
\hline \multicolumn{3}{|c|}{ Geburtsland der Eltern (Ref.: Deutschland) } \\
\hline \multirow[t]{2}{*}{1 Elternt. Ausland } & $-0,180 * *$ & 0,013 \\
\hline & $(0,024)$ & $(0,025)$ \\
\hline \multirow[t]{2}{*}{2 Eltern Ausland } & $-0,148 * *$ & 0,038 \\
\hline & $(0,045)$ & $(0,043)$ \\
\hline \multirow[t]{2}{*}{ Junge (Ref.: Mädchen) } & $-0,060 * *$ & $-0,055 * *$ \\
\hline & $(0,016)$ & $(0,014)$ \\
\hline \multicolumn{3}{|l|}{ Geschwister (Ref.: keine) } \\
\hline \multirow[t]{2}{*}{1 Geschwister } & 0,023 & 0,004 \\
\hline & $(0,020)$ & $(0,018)$ \\
\hline \multirow[t]{2}{*}{ mind. 2 Geschwister } & $-0,078 * *$ & $-0,060 * *$ \\
\hline & $(0,024)$ & $(0,021)$ \\
\hline \multicolumn{3}{|l|}{ Erhebungsjahr (Ref.: 1997) } \\
\hline \multirow[t]{2}{*}{2003} & $0,070 * *$ & $-0,001$ \\
\hline & $(0,025)$ & $(0,022)$ \\
\hline \multirow[t]{2}{*}{2007} & $0,083 * *$ & $-0,015$ \\
\hline & $(0,026)$ & $(0,024)$ \\
\hline \multirow[t]{2}{*}{2011} & $0,110 * *$ & 0,011 \\
\hline & $(0,027)$ & $(0,024)$ \\
\hline \multirow[t]{2}{*}{ Konstante } & & $0,354 * *$ \\
\hline & & $(0,029)$ \\
\hline
\end{tabular}


Tab. 2 (Fortsetzung)

\begin{tabular}{lll}
\hline & Bruttoeffekte & Modell 1 \\
\hline Fallzahl(Jugendliche) & 3622 & 3622 \\
Anzahl an Kreisen & 308 & 308 \\
$\mathrm{R}^{2}$ (within) & $/$ & 0,237 \\
\hline
\end{tabular}

Ref. Referenzkategorie

Signifikanzniveaus: $+p<0,10 ;{ }^{*} p<0,05 ;{ }^{*} p<0,01$; robuste Standardfehler in Klammern

${ }^{a}$ Mittelwert zentriert

weshalb eine besondere Wertschätzung protestantischer Werte und höhere Wahrscheinlichkeit der Vermittlung dieser in der Sozialisation nicht gestützt werden kann.

Als zweite Strategie verwenden wir einen Interaktionsterm für den Besuch religiöser Veranstaltungen (Kirchgang, Moscheebesuch) mit der Religionszugehörigkeit. Protestantische Werte sollten bei häufigerem Kirchgang ausgeprägter sein. Bei Muslimen könnten hingegen häufige Besuche religiöser Veranstaltungen die eigene Religionszugehörigkeit als „Bright Boundary“ verstärken und somit mit geringeren Bildungserfolgen einhergehen.

Modell 3, Tab. 3, enthält zunächst die Haupteffekte für den Besuch religiöser Veranstaltungen. Eine mindestens monatliche Teilnahme hieran erhöht die Gymnasialchancen der Jugendlichen um 5\%-Punkte gegenüber jenen mit Müttern, die nie an solchen Veranstaltungen teilnehmen. Mit den Interaktionseffekten in Modell 4 wird jetzt die Abweichung z. B. von evangelischen Müttern mit seltenem Kirchgang $(0,054)$ und mindestens monatlichem Kirchgang $(-0,019)$ von katholischen Müttern mit gleicher Kirchganghäufigkeit ausgewiesen. Diese Abweichungen sind nicht signifikant und zeigen im Fall des Interaktionseffekts für mindestens monatlichen Kirchgang und protestantische Zugehörigkeit zudem nicht in die erwartete Richtung eines positiven Effekts. Die Interaktionseffekte für muslimische Mütter sind samt alle negativ, wenn auch nicht signifikant. Könnte dies trotzdem ein Hinweis auf „Bright Boundaries“ als Hindernis für schulischen Erfolg sein? Wir sind hier skeptisch, denn die negativen Interaktionseffekte heben lediglich die positiven Haupteffekte für Besuch religiöser Veranstaltungen auf. Das heißt, bei muslimischen Familien gibt es keinen Zusammenhang zwischen Häufigkeit des Moscheebesuchs und Gymnasialbeteiligung.

\subsection{Befunde zur Gymnasialbeteiligung in der Diaspora}

Nach Hypothese $H 1$ ist das Erfolgsstreben von Angehörigen religiöser Gemeinschaften ausgeprägter, wenn sie sich in einer Minderheit befinden. Deshalb präsentieren wir in Tab. 4 Anteile an Gymnasialbesuchen nach Religionszugehörigkeit der Mutter und konfessioneller Prägung des Wohnkreises, wobei wir uns an die Befunde zu Westdeutschland in den Nachkriegsjahrzehnten anschließend auf den Anteil an Katholiken im Kreis konzentrieren. Die Einteilung zur konfessionellen Prägung unterscheidet nach Kreisen mit weniger als $15 \%$, mit 15 bis unter $25 \%$, mit 25 bis unter $35 \%$, mit 35 bis unter $45 \%$, mit 45 bis unter $55 \%$ und mindestens $55 \%$ Katholiken. 
Tab. 3 Zur Bedeutung von Religion für den Gymnasialbesuch - Lineare Wahrscheinlichkeitsmodelle mit fixen Effekten für Kreise. (Quellen: SOEP, v29; eigene Berechnungen)

\begin{tabular}{|c|c|c|c|}
\hline & Modell 2 & Modell 3 & Modell 4 \\
\hline \multicolumn{4}{|c|}{ Religion der Mutter (Ref.: kath.) } \\
\hline Evangelisch & $\begin{array}{l}-0,018 \\
(0,028)\end{array}$ & $\begin{array}{l}-0,024 \\
(0,021)\end{array}$ & $\begin{array}{l}-0,041 \\
(0,034)\end{array}$ \\
\hline Muslimisch & $\begin{array}{l}-0,016 \\
(0,041)\end{array}$ & $\begin{array}{l}-0,052 \\
(0,037)\end{array}$ & $\begin{array}{l}-0,017 \\
(0,057)\end{array}$ \\
\hline Sonstige & $\begin{array}{l}-0,043 \\
(0,045)\end{array}$ & $\begin{array}{l}-0,085^{*} \\
(0,042)\end{array}$ & $\begin{array}{l}-0,108 \\
(0,073)\end{array}$ \\
\hline Konfessionslos & $\begin{array}{l}-0,038 \\
(0,033)\end{array}$ & $\begin{array}{l}-0,047 \\
(0,031)\end{array}$ & $\begin{array}{l}-0,029 \\
(0,037)\end{array}$ \\
\hline \multicolumn{4}{|l|}{ Religiös homogam } \\
\hline Katholisch & $\begin{array}{l}0,062^{*} \\
(0,029)\end{array}$ & & \\
\hline Evangelisch & $\begin{array}{l}0,047^{+} \\
(0,028)\end{array}$ & & \\
\hline \multicolumn{4}{|c|}{ Kirchgang, Moscheebesuch etc. der Mutter (Ref.: nie) } \\
\hline Seltener & & $\begin{array}{l}0,028 \\
(0,019)\end{array}$ & $\begin{array}{l}0,013 \\
(0,032)\end{array}$ \\
\hline Mind. monatlich & & $\begin{array}{l}0,050^{*} \\
(0,024)\end{array}$ & $\begin{array}{l}0,070^{+} \\
(0,036)\end{array}$ \\
\hline \multicolumn{4}{|l|}{$\begin{array}{l}\text { Interaktionsterme } \\
\text { Seltener Kirchgang }\end{array}$} \\
\hline *Evangelisch $(n=644)$ & & & $\begin{array}{l}0,054 \\
(0,042)\end{array}$ \\
\hline *Muslimisch $(n=68)$ & & & $\begin{array}{l}-0,011 \\
(0,072)\end{array}$ \\
\hline *Sonstige $(n=37)$ & & & $\begin{array}{l}0,096 \\
(0,115)\end{array}$ \\
\hline *Konfessionslos $(n=60)$ & & & $\begin{array}{l}-0,111 \\
(0,077)\end{array}$ \\
\hline \multicolumn{4}{|l|}{ Mind. monatlicher Kirchgang } \\
\hline *Evangelisch $(n=288)$ & & & $\begin{array}{l}-0,019 \\
(0,050)\end{array}$ \\
\hline *Muslimisch $(n=79)$ & & & $\begin{array}{l}-0,101 \\
(0,075)\end{array}$ \\
\hline *Sonstige $(n=122)$ & & & $\begin{array}{l}-0,005 \\
(0,090)\end{array}$ \\
\hline *Konfessionslos $(n=11)$ & & & $\begin{array}{l}0,040 \\
(0,168)\end{array}$ \\
\hline Fallzahl(Jugendliche) & 3619 & 3622 & 3622 \\
\hline Anzahl an Kreisen & 308 & 308 & 308 \\
\hline $\mathrm{R}^{2}$ (within) & 0,239 & 0,238 & 0,241 \\
\hline
\end{tabular}

Robuste Standardfehler in Klammern; $\mathrm{n}=$ Fallzahlen

Unter Kontrolle von hochkulturellen Aktivitäten der Mutter, höchstem Bildungsabschluss u. ISEI, alleinerziehend, Befragungsteilnahme Vater, Migrationshintergrund, Geschwistern, Geschlecht, Erhebungsjahr (siehe Modell 1, Tab. 2)

Ref. Referenzkategorie

$+p<0,10 * p<0,05 ; * * p<0,01$ 
Tab. 4 Gymnasialquoten nach Religionszugehörigkeit der Mutter und Anteil an Katholiken im Wohnkreis. (Quellen: SOEP, v29; Forsa-Bus 1997-2007; eigene Berechnungen)

\begin{tabular}{lllllllllllll}
\hline Kreis & $<15 \%$ & $\begin{array}{l}15 \% \\
-<25 \%\end{array}$ & $\begin{array}{l}25 \% \\
-<35 \%\end{array}$ & $\begin{array}{l}35 \% \\
-<45 \%\end{array}$ & $\begin{array}{l}45 \% \\
-<55 \%\end{array}$ & $\geq 55 \%$ \\
\hline Religion (Mutter) & $\begin{array}{l}\text { Gym } \\
(\%)\end{array}$ & $n$ & $\begin{array}{l}G y m \\
(\%)\end{array}$ & $n$ & $\begin{array}{l}G y m \\
(\%)\end{array}$ & $n$ & $\begin{array}{l}G y m \\
(\%)\end{array}$ & $n$ & $\begin{array}{l}G y m \\
(\%)\end{array}$ & $n$ & $\begin{array}{l}G y m \\
(\%)\end{array}$ & $n$ \\
\hline Katholisch & 48 & 60 & 44 & 115 & 44 & 206 & 47 & 206 & 37 & 235 & 29 & 576 \\
Evangelisch & 38 & 377 & 36 & 252 & 38 & 265 & 47 & 180 & 46 & 121 & 33 & 153 \\
Muslimisch & 25 & 32 & 18 & 44 & 11 & 79 & 6 & 54 & $(4)$ & 26 & $(8)$ & 24 \\
Sonstige & 17 & 46 & 11 & 44 & 46 & 37 & $(48)$ & 23 & $(23)$ & 22 & $(18)$ & 17 \\
Konfessionslos & 36 & 124 & 52 & 66 & 35 & 69 & 31 & 45 & 36 & 50 & 31 & 70 \\
\hline Insgesamt & 37 & 639 & 36 & 521 & 37 & 656 & 41 & 508 & 37 & 454 & 29 & 840 \\
\hline
\end{tabular}

Anteil des Gymnasialbesuchs bei weniger als 30 Beobachtungen in Klammern

Gym Gymnasialquote, $n$ Fallzahlen

Tab. 5 Gymnasialquoten nach Religionszugehörigkeit der Mutter und Bevölkerungsdichte des Kreises. (Quellen: SOEP, v29; eigene Berechnungen)

\begin{tabular}{|c|c|c|c|c|c|c|c|c|c|c|}
\hline \multirow{3}{*}{$\begin{array}{l}\mathrm{X} \text { Einw. } / \mathrm{km}^{2} \\
\text { Religion (Mutter) }\end{array}$} & \multicolumn{2}{|c|}{ 1. Q. (= dünn) } & \multicolumn{2}{|c|}{ 2. Quintil } & \multicolumn{2}{|c|}{ 3. Quintil } & \multicolumn{2}{|c|}{ 4. Quintil } & \multicolumn{2}{|c|}{ 5. Q. (=dicht $)$} \\
\hline & \multicolumn{2}{|c|}{$\mathrm{X} \leq 120$} & \multicolumn{2}{|c|}{$120<X \leq 179$} & \multicolumn{2}{|c|}{$179<X \leq 301$} & \multicolumn{2}{|c|}{$301<X \leq 970$} & \multicolumn{2}{|c|}{$X>970$} \\
\hline & $\begin{array}{l}\text { Gym } \\
(\%)\end{array}$ & $n$ & $\begin{array}{l}\text { Gym } \\
(\%)\end{array}$ & $n$ & $\begin{array}{l}\text { Gym } \\
(\%)\end{array}$ & $n$ & $\begin{array}{l}\text { Gym } \\
(\%)\end{array}$ & $n$ & $\begin{array}{l}\text { Gym } \\
(\%)\end{array}$ & $n$ \\
\hline Katholisch & 26 & 213 & 35 & 233 & 34 & 323 & 37 & 346 & 52 & 285 \\
\hline Evangelisch & 35 & 185 & 28 & 212 & 40 & 295 & 40 & 343 & 47 & 314 \\
\hline Muslimisch & (0) & 2 & (14) & 28 & 13 & 39 & 12 & 86 & 12 & 104 \\
\hline Sonstige Rel. & (0) & 14 & (17) & 23 & 24 & 38 & 28 & 64 & 36 & 50 \\
\hline Konfessionslos & 35 & 52 & 31 & 51 & 32 & 80 & 39 & 95 & 41 & 147 \\
\hline Insgesamt & 30 & 466 & 30 & 547 & 35 & 775 & 35 & 934 & 43 & 900 \\
\hline
\end{tabular}

Anteil des Gymnasialbesuchs bei weniger als 30 Beobachtungen in Klammern Gym Gymnasialquote, $n$ Fallzahlen

Die Gymnasialquoten fallen für Jugendliche mit einer katholischen Mutter besonders niedrig aus, wenn der Anteil an Katholiken in einem Kreis 45\% und mehr beträgt. Jugendliche mit einer evanglischen Mutter weisen hingegen besonders hohe Quoten auf, wenn der Anteil an Katholiken zwischen 35 und 55\% liegt. Es lässt sich zudem erkennen, dass mit steigendem Anteil an Katholiken in einem Kreis der Vorsprung von Katholiken gegenüber Protestanten sinkt und sich bis hin zu Nachteilen verkehrt. Die Differenzen betragen über die sechs Kategories +10, +8, +6, 0, -9 und schließlich -4\%-Punkte.

Auch bei den Muslimen sehen wir mit zunehmendem Anteil an Katholiken in einem Kreis eine abnehmende Gymnasialbeteiligung. Da sich Muslime in Deutschland immer in einer Minderheitensituation befinden, war das nicht unbedingt zu erwarten. Diese Zahlen, wie auch die insgesamt sinkenden Gymnasialquoten ab einem Anteil von $55 \%$ Katholiken in einem Kreis, könnten auch auf Stadt-LandUnterschieden beruhen. In Westdeutschland ist der Anteil an konfessionell Gebundenen in ländlichen Regionen höher als in städtischen Gebieten. Bei einer niedrigen Bevölkerungsdichte könnte zugleich das Angebot an Gymnasien geringer und deren Erreichbarkeit schlechter sein. Deshalb stellen wir die Gymnasialquoten auch nach der Bevölkerungsdichte von Kreisen vor (siehe Tab. 5). 
In dünn besiedelten Kreisen sind die Gymnasialquoten von Katholiken besonders niedrig, in dicht besiedelten dafür überdurchschnittlich hoch. Diese Variation über die Bevölkerungsdichte ist bei Katholiken ausgeprägter als bei Protestanten. Besonders in dünn besiedelten Kreisen haben Katholiken geringere Gymnasialquoten als Protestanten (-9\%-Punkte). Bei Kindern von muslimischen Müttern lassen sich keine Variationen über die Bevölkerungsdichte erkennen.

Modelle mit festen Effekten für Kreise erlauben es nicht, die konfessionelle Prägung eines Kreises als gesonderte Kovariate aufzunehmen. Es können aber sogennante Cross-level-Interaktionsterme zwischen der Konfessionszugehörigkeit der Mutter und der konfessionellen Prägung modelliert werden. Damit die Modelle übersichtlich und die Haupt- und Interaktionseffekte einer Interpretation zugänglich bleiben, beschränken wir uns auf Jugendliche mit einer katholischen oder protestantischen Mutter. Modell 5a und 5b, Tab. 6, enthalten den Haupteffekt für protestantische Mütter und den Interaktionseffekt zwischen diesen Müttern und dem Anteil an Katholiken ohne und mit den bekannten Kontrollvariablen (vgl. Modell 1, Tab. 2). In beiden Modellen werden zwei gegenläufige Effekte ausgewiesen, wonach sich der Nachteil von Protestanten mit steigendem Anteil an Katholiken aufhebt. Werden Kontrollvariablen berücksichtigt, ist nur noch der Haupteffekt signifikant (vgl. Modell 5b). Werden nun aber Haupt- und Interaktionseffekt miteinander verrechnet, sind diese Werte auf dem 5\%-Niveau statistisch signifikant und negativ, wenn der Anteil an Katholiken z. B. 10, 20, 30 oder 40\% beträgt.

Ob diese Variation auf Unterschiede in der Siedlungsdichte zurückzuführen ist, wird mit den Modellen 6 und 7 geprüft. Nach Modell 6 sinkt der Vorteil für Protestanten mit steigender Bevölkerungsdichte. Werden der Interaktionsterm zum Anteil an Katholiken und zur Bevölkerungsdichte gleichzeitig in der Modellierung berücksichtigt (vgl. Modell 7), sinken die dazugehörigen Koeffizienten im Vergleich zu Modell 5 b und 6 und sind dann beide nicht signifikant. Somit lässt sich nicht abschließend beurteilen, ob der Katholikenanteil oder die Bevölkerungsdichte relevant(er) ist.

Es stellt sich aber auch die Frage, ob die Minderheitensituation tatsächlich zu einem höheren Erfolgsstreben führt oder ob räumliche Mobilität und unbeobachtete Familienmerkmale dafür verantwortlich sind (vgl. Alternativhypothese $H 2$ ). Um dies zu testen, teilen wir die Stichprobe in die Mütter auf, die am Ort der Kindheit oder einem anderen Ort leben (zum Verlust von Fällen vgl. die Erläuterungen am Ende von Abschn. 6.1). Für die Sesshaften werden keine signifikanten Effekte zwischen protestantischer Mutter und der konfessionellen Prägung des Kreises ausgewiesen (Modell 5c). Wir sehen aber ausgeprägte, auf dem 1\%-Niveau signifikante Hauptund Interaktionseffekte in der Gruppe der mobilen Mütter (Modell 5d). Dies sind sehr deutliche Hinweise darauf, dass nicht die religiöse Diaspora, sondern unbeobachtete Individualmerkmale, die über den formalen Bildungsstand und die soziooekonomische Position der Eltern hinausgehen für Unterschiede im Bildungserfolg verantwortlich sind.

Als Alternativmodellierung wird ein Vergleich der konfessionellen Prägung von Geburts- und aktuellem Wohnkreis vorgenommen (zum Fallzahlverlust vgl. erneut Abschn. 6.1). Wir sind uns bewusst, dass die Population, für die gültige Angaben vorliegen, möglicherweise selektiv ist und dass die Definition von „Umzug in Diaspora“ und „Auszug aus Diaspora“ suboptimal ist. In Ermangelung an Alternativen und um 
Tab. 6 Gymnasialbesuch von Protestanten und Katholiken in Abhängigkeit von Diaspora, Bevölkerungsdichte und innerdeutscher Mobilität - Lineare Wahrscheinlichkeitsmodelle mit fixen Effekten für Kreise. (Quellen: SOEP, v29; Forsa-Bus 1997-2007; eigene Berechnungen)

\begin{tabular}{|c|c|c|c|c|c|c|c|c|c|c|}
\hline & Modell 5a & Modell 5b & Modell 6 & Modell 7 & Modell 5c & Modell 5d & Modell 8a & Modell 8b & Modell 9a & Modell 9b \\
\hline & Ohne Kontrolle & & & & Sesshaft & Mobil & Ohne Kontrolle & & Ohne Kontrolle & \\
\hline \multicolumn{11}{|l|}{ Religion Mutter (Ref.: kath.) } \\
\hline Evangelisch & $\begin{array}{l}-0,127^{+} \\
(0,067)\end{array}$ & $\begin{array}{l}-0,119^{*} \\
(0,058)\end{array}$ & $\begin{array}{l}0,132 \\
(0,086)\end{array}$ & $\begin{array}{l}0,056 \\
(0,127)\end{array}$ & $\begin{array}{l}0,094 \\
(0,095)\end{array}$ & $\begin{array}{l}-0,328 * * \\
(0,104)\end{array}$ & $\begin{array}{l}-0,019 \\
(0,042)\end{array}$ & $\begin{array}{l}-0,000 \\
(0,036)\end{array}$ & $\begin{array}{l}-0,027 \\
(0,042)\end{array}$ & $\begin{array}{l}-0,008 \\
(0,037)\end{array}$ \\
\hline \multicolumn{11}{|l|}{ Kreisanteil Katholiken } \\
\hline *Evangelisch & $\begin{array}{l}0,269^{+} \\
(0,152)\end{array}$ & $\begin{array}{l}0,191 \\
(0,130)\end{array}$ & & $\begin{array}{l}0,112 \\
(0,140)\end{array}$ & $\begin{array}{l}-0,275 \\
(0,215)\end{array}$ & $\begin{array}{l}0,646^{* *} \\
(0,220)\end{array}$ & & & & \\
\hline \multicolumn{11}{|l|}{ Bevölkerungsdichte (ln) } \\
\hline *Evangelisch & & & $\begin{array}{l}-0,030 * \\
(0,015)\end{array}$ & $\begin{array}{l}-0,024 \\
(0,016)\end{array}$ & & & & & & \\
\hline \multicolumn{11}{|c|}{ Geburtsort Mutter-aktueller Wohnort (Ref.: sesshaft) } \\
\hline Umzug in Diaspora $(n=51)$ & & & & & & & $\begin{array}{l}0,272 * * \\
(0,085)\end{array}$ & $\begin{array}{l}0,123 \\
(0,078)\end{array}$ & & \\
\hline Auszug aus Diaspora $(n=40)$ & & & & & & & $\begin{array}{l}0,030 \\
(0,099)\end{array}$ & $\begin{array}{l}-0,019 \\
(0,077)\end{array}$ & & \\
\hline Sonstige Mobile $(n=632)$ & & & & & & & $\begin{array}{l}0,039 \\
(0,040)\end{array}$ & $\begin{array}{l}-0,033 \\
(0,037)\end{array}$ & & \\
\hline \multicolumn{11}{|l|}{ Distanz $($ Ref.: $<50 \mathrm{~km})$} \\
\hline $50-<100(n=140)$ & & & & & & & & & $\begin{array}{l}0,069 \\
(0,069)\end{array}$ & $\begin{array}{r}-0,005 \\
(0,053)\end{array}$ \\
\hline $100-<200(n=107)$ & & & & & & & & & $\begin{array}{c}0,200 * * \\
(0,069)\end{array}$ & $\begin{array}{l}0,077 \\
(0,067)\end{array}$ \\
\hline $200-<300(n=60)$ & & & & & & & & & $\begin{array}{c}0,239 * * \\
(0,084)\end{array}$ & $\begin{array}{l}0,117 \\
(0,075)\end{array}$ \\
\hline$\geq 300(n=87)$ & & & & & & & & & $\begin{array}{l}0,081 \\
(0,069)\end{array}$ & $\begin{array}{r}-0,002 \\
(0,064)\end{array}$ \\
\hline
\end{tabular}


Tab. 6 (Fortsetzung)

\begin{tabular}{|c|c|c|c|c|c|c|c|c|c|c|}
\hline & Modell 5a & Modell 5b & Modell 6 & Modell 7 & \multirow{2}{*}{$\begin{array}{l}\text { Modell 5c } \\
\text { Sesshaft }\end{array}$} & \multirow{2}{*}{$\begin{array}{l}\text { Modell 5d } \\
\text { Mobil }\end{array}$} & Modell 8a & Modell 8b & Modell 9a & Modell 9b \\
\hline & Ohne Kont & & & & & & \multicolumn{2}{|l|}{ Ohne Kontrolle } & \multicolumn{2}{|l|}{ Ohne Kontrolle } \\
\hline Fallzahl(Jugendliche) & 2746 & 2746 & 2746 & 2746 & 1420 & 1029 & 1371 & 1371 & 1395 & 1395 \\
\hline Anzahl an Kreisen & 301 & 301 & 301 & 301 & 273 & 249 & 270 & 270 & 273 & 273 \\
\hline $\mathrm{R}^{2}$ (within) & 0,002 & 0,245 & 0,245 & 0,245 & 0,242 & 0,242 & 0,011 & 0,223 & 0,02 & 0,228 \\
\hline
\end{tabular}

Robuste Standardfehler in Klammern; $\mathrm{n}=$ Fallzahlen

Unter Kontrolle von hochkulturellen Aktivitäten der Mutter, höchstem Bildungsabschluss u. ISEI, alleinerziehend, Befragungsteilnahme Vater, Migrationshintergrund, Geschwistern, Geschlecht, Erhebungsjahr (siehe Modell 1, Tab. 2)

Ref. Referenzkategorie

$+p<0,10 * p<0,05 ; * * p<0,01$ 
weitere Hinweise zur Gültigkeit des Arguments zu unbeobachteten Aspirationen der Mutter zu erhalten, präsentieren wir Modell 8a ohne und Modell 8b mit Kontrollvariablen. Jugendliche mit Müttern, die in eine Diaspora gezogen sind, haben ohne Kontrolle weiterer Variablen eine um 27,2 und mit Kontrolle eine um 12,3\%-Punkte höhere Wahrscheinlichkeit eines Gymnasialbesuchs als Sesshafte, wobei in dem Modell mit Kontrollvariablen der Effekt für „Umzug in die Diaspora“ statistisch nicht mehr signifikant ist. Mit 51 Beobachtungen ist die Fallzahl jedoch auch sehr gering. Für die Variable „Auszug aus der Diaspora“ und für „sonstige Mobile“ beobachten wir keine entsprechenden Effekte. Mit und ohne Kontrolle von Kovariaten sind die geschätzten Effekte nahe Null. Mobilität der Mutter scheint nicht automatisch mit höherem Bildungserfolg des Kindes einherzugehen, sondern nur Umzüge mit einem starken Wechsel des Umfelds und damit möglicherweise größeren Umzugsdistanzen. Deshalb testen wir zusätzlich noch einmal den Zusammenhang von Mobilität der Mutter und Bildungserfolg ihres Kindes, indem wir Umzüge zwischen Kreisen nach ihrer Entfernung erfassen. Bei Umzügen mit einer Distanz von 100 bis 200 sowie 200 bis $300 \mathrm{~km}$ Luftlinie werden um 20 bzw. 23,9\%-Punkte höhere Gymnasialquoten für die Jugendlichen geschätzt (Modell 9a). Erstaunlich ist, dass bei Umzügen von über $300 \mathrm{~km}$ der positive Effekt deutlich kleiner ausfällt und trotz vergleichbarer Fallzahlen statistisch nicht signifikant ist. Nach Berücksichtigung der Kontrollvariablen reduzieren sich die Effekte erheblich und sind allesamt nicht mehr signifikant (Modell 9b).

Exkurs zu Ostdeutschland. Bisher haben wir uns nur mit Westdeutschland beschäftigt. Falls es Diaspora-Effekte in Deutschland gibt, wäre zu erwarten, dass diese sich insbesondere in Ostdeutschland zeigen. „Von den 90 Prozent der Ostdeutschen [sic] die 1953 noch Mitglieder in einer der christlichen Konfessionen waren, blieben 1989 gerade einmal 27 Prozent übrig. Dies ging vor allem zu Lasten der evangelischen Kirche, fiel doch deren Mitgliedschaft von 80 Prozent der Bevölkerung auf dem Gebiet der neuen Bundesländer auf ca. 25 Prozent 1989. Die katholische Kirche schrumpfte im gleichen Zeitraum von elf auf ungefähr 4 Prozent" (Pickel 2013, S. 79). Nach dem Zusammenbruch der DDR kam es zu keiner Revitalisierung der Konfessionsmitgliedschaften in den neuen Bundesländern (Pickel 2013). Zudem bedeutet Konfessionslosigkeit in Ostdeutschland viel häufiger als in Westdeutschland keine religiöse Praxis und keine religiösen Überzeugungen zu haben (Wohlrab-Sahr 2009, S. 154).

Für Ostdeutschland können wir keine Modelle mit festen Effekten für Kreise schätzen, weil wir in unserem Zuschnitt der SOEP-Daten für Ostdeutschland nur 54 Jugendliche mit einer katholischen Mutter beobachten und diese sich auf nur wenige Kreise verteilen. Zudem gab es in den letzten Jahren in mehreren neuen Bundesländern Kreisreformen. Weiterhin ist die Gruppe der nicht-christlichen Religionsangehörigen in der Stichprobe zahlenmäßig kaum vertreten und muss daher von vornherein aus den Analysen ausgeschlossen werden.

Wenn wir uns die Gymnasialquoten von Jugendlichen mit katholischen, protestantischen und konfessionslosen Müttern anschauen und ein „einfaches“ Regressionsmodell ohne Kovariaten schätzen (siehe Modell A1, Tab. 7 im Anhang), dann haben wir in eben dieser Reihenfolge Quoten von 52, 46 und 36\%. Die Differenz zwischen Katholiken und Konfessionslosen ist wegen der geringen Fallzahl „nur“ auf dem 
10\%-Niveau, die zwischen Protestanten und Konfessionslosen auf dem 5\%-Niveau signifikant.

Werden die gleichen Variablen wie in Modell 1, Tab. 2, zur Kontrolle der sozialen Lage der Familie, ihrer sozio-demographischen Struktur und des Erhebungsjahrs berücksichtigt, bleiben die Effekte für die Konfessionszugehörigkeit in Ostdeutschland nahezu unverändert (siehe Modell A2, Tab. 7 im Anhang). Die Kinder, die in der größten Gruppe, in der Gruppe der Konfessionslosen aufwachsen, haben die geringsten Gymnasialquoten, gefolgt von Kindern der zweitgrößten Gruppe, die der Protestanten, und die höchsten Werte weist die kleinste Gruppe, nämlich die der Katholiken auf. Der Unterschied zwischen Protestanten und Katholiken ist nicht signifikant, aber der Unterschied zwischen Katholiken und Konfessionslosen sowie zwischen Protestanten und Konfessionslosen ist jeweils auf dem 10\%-Niveau signifikant.

\section{Fazit: Keine Differenzen im Gymnasialbesuch zwischen Katholiken, Protestanten und Muslimen in Westdeutschland}

Wir sind angetreten mit dem Ziel, aktuelle Befunde zu liefern, ob und, wenn ja, wieso es Differenzen im Bildungserfolg nach Religionszugehörigkeit der Eltern in Westdeutschland gibt. Hierzu haben wir Webers Ausführungen zur protestantischen Ethik vorgestellt sowie Becker und Wößmanns (2009) Alternativerklärung zum Lesegebot und damit einer (historisch) früher einsetzenden umfänglichen Alphabetisierung von Protestanten im Vergleich zu Katholiken (zumindest in Preußen). Ob Protestanten und Katholiken sich heute noch in ihrem Arbeitsethos unterscheiden und dies möglicherweise zu differentiellen Bildungserfolgen führt, haben wir zunächst vor dem Hintergrund bisheriger Forschungsarbeiten diskutiert. Nach Schaltegger und Torgler (2010), die Daten der European Value Study für 16 Länder ausgewertet haben, gibt es diese Differenzen. Mithilfe des Ansatzes von Alba zu muslimischer Religionszugehörigkeit als „Bright Boundary“ in Deutschland (und anderen westeuropäischen Ländern) und Ausführungen zum ,Stereotype Threat“ haben wir argumentiert, wieso bei Muslimen geringere Bildungserfolge zu erwarten sind.

Die beiden Forschungsfragen zu religionsspezifischen Differenzen im Bildungserfolg ließen sich mit den SOEP-Daten nicht bestätigen. Jugendliche mit einer evangelischen Mutter haben keine höheren Gymnasialquoten als Jugendliche mit einer katholischen (Forschungsfrage 1) und Jugendliche aus muslimischen Familien haben nach Kontrolle sozialstruktureller Merkmale ebenfalls keine statistisch signifikant geringeren Gymnasialquoten als Jugendliche aus Familien, die einer der beiden christlichen Großkirchen angehören (Forschungsfrage 2). Auch alternative Prüfungen, um die Heterogenität innerhalb der Religionsgruppen besser zu erfassen, lieferten keine Hinweise auf religionsspezifische Differenzen. Protestantische Homogamie steigert im Vergleich zu katholischer Homogamie nicht die Chance, in der Sekundarstufe ein Gymnasium zu besuchen, und mit häufigerem Kirchgang von Protestanten, der mit einer stärkeren Verinnerlichung protestantischer Werte einhergehen sollte, sind die Chancen auch nicht größer. Ebenso scheint ein häufiger Moscheebesuch, die „Bright Boundary“ zwischen der muslimischen Religionszugehörigkeit und der christlich geprägten Mehrheitsgesellschaft nicht dergestalt zu stärken, dass sich der 
Bildungserfolg der Kinder verringert. Somit kann festgehalten werden, dass Kinder aus evangelischen, katholischen und muslimischen Familien vergleichbare Gymnasialquoten haben.

Zur Prüfung der Diaspora-Effekte (Hypothese H1) ist zunächst anzumerken, dass sich Kinder aus muslimischen Familien in Deutschland in einer Minderheitensituation befinden. Würden die Argumente zutreffen, dass ein Zusammenrücken, eine stärkere Kontrolle und/oder eine höhere Motivation zu höherem Bildungserfolg führen, hätte sich dies bei den Muslimen bereits bei den zuvor berichteten Beteiligungsquoten zeigen müssen. Die differenzierte Betrachtung von Diaspora-Effekten bei Mitgliedern der beiden christlichen Großkirchen ergab, dass der Nachwuchs von Katholiken im Vergleich zu dem von Protestanten besonders häufig ein Gymnasium besucht, wenn dieser sich in einer regionalen Minderheitensituation befindet, d. h. wenn im gleichen Kreis wenige Katholiken wohnen. In multivariaten Modellen zeigten sich diese Effekte zwar auch, dort aber nicht mehr so deutlich wie in den trivariaten Tabellen und zudem inferenzstatistisch nicht abgesichert. Die Modelle auf Basis von Substichproben zu sesshaften und zu mobilen Müttern haben ergeben, dass nur bei mobilen Müttern die Interaktion von eigener Konfessionszugehörigkeit und konfessioneller Prägung des Kreises mit dem Bildungserfolg korreliert.

Bei Protestanten und Katholiken, die in einen Kreis zugewandert sind, der mehrheitlich anders geprägte ist als ihre eigene Konfession, handelt es sich mutmaßlich um eine Selektion von Personen, die Eigenschaften aufweisen, die auch den Bildungserfolg des Kindes beeinflussen (vgl. Alternativhypothese H2). Hierbei könnte es sich z. B. um Eltern mit akademischen Abschlüssen handeln, die berufsbedingt in eine konfessionell anders geprägt Umgebung gezogen sind. Wie aus der Forschung hinlänglich bekannt, besucht der Nachwuchs von Akademikereltern häufiger weiterführende Bildungseinrichtungen. Zumindest für die Merkmale höchste Bildung und sozio-oekonomischer Status der Eltern haben wir kontrolliert. Darüber hinaus müsste es noch weitere Merkmale geben, die sowohl die Wanderung als auch den Bildungserfolg des Kindes beeinflussen. Es wurden positive Befunde für Umzüge in die Diaspora auf höheren Bildungserfolg ausgewiesen. Einschränkend ist darauf hinzuweisen, dass in den multivariaten Modellen dieser Effekt statistisch nicht signifikant ist. Dies mag aber auch an der geringen Zahl der Fälle liegen. Problematisch an dieser Interpretation ist auch, dass die reine Umzugsdistanz, die ebenso gut ein Indikator für berufliche Ambitionen und Bereitwilligkeit des Verlassens des angestammten Umfelds sein sollte, keine signifikanten Ergebnisse liefert, wenn für die sozial-strukturelle Lage der Familie kontrolliert wird.

Wir beobachten nahezu keine Differenzen im Bildungserfolg zwischen Schülern aus protestantischem und katholischem Elternhaus in Westdeutschland. Das heißt aber nicht, dass wir damit Webers (2006) Erklärungen zur protestantischen Ethik als widerlegt ansehen. Möglicherweise waren sie in seiner Zeit gültig, sind aber heute nicht mehr relevant. Wir widerlegen auch nicht die Argumente zum Lesegebot (Becker und Wößmann 2009, 2010; Durkheim 1983). Historisch gesehen mag es deshalb zu Unterschieden in der Ausbreitung von Schulen und Alphabetisierung gekommen sein. Nur heute, nach einer längeren Phase des Ausbaus des Bildungssystems, der zunehmenden Rationalisierung und der starken Bedeutung von Lesekompetenzen für Lebenschancen, gibt es offenbar keine konfessionellen Unterschiede. 
Was wir beobachten, sind Hinweise auf mögliche Diaspora-Effekte, zumindest für Katholiken und Protestanten. Die von uns diskutierten Erklärungen beziehen sich auf stärkere Kontrolle durch die Gemeinschaft in der Minderheitensituation und besonders hohe Motivation, sich in der (regionalen) Mehrheitsgesellschaft zu behaupten, aus dem ein starkes Bildungsstreben und ein größerer Bildungserfolg ihrer Nachkommen resultiert. Die Befunde stützen teilweise eine dritte Erklärung: selektive Mobilitätsprozesse der Eltern. Allerdings erbringen verschiedene Modellierungsstrategien keine konsistent belastbaren Ergebnisse. Die Befunde liefern über die eigentliche Frage hinaus einen sehr wichtigen Beitrag zur Erforschung der Wirkung von Kontexten in der Bildungsforschung. Wir beobachten nur eine Relevanz des regionalen Kontextes, wenn wir uns auf die Eltern beschränken, die nicht mehr am Ort der Kindheit leben. Dies lässt darauf schließen, dass nicht der Kontext relevant ist, sondern individuelle Merkmale der Eltern. (Unbeobachtete) Selektionsprozesse in der räumlichen Mobilität und der Schulwahl sind zentrale Herausforderungen in der Identifizierung z. B. von Nachbarschafts- oder Schulkontexteffekten. Wir haben eine Möglichkeit präsentiert, die sich zumindest für die Identifizierung von regionalen Kontexteffekten eignet.

In unserem kleinen Exkurs zu Ostdeutschland, wo Mitglieder von Religionsgemeinschaften eine Minderheit darstellen, haben wir sowohl für Katholiken als auch für Protestanten deutlich höhere Gymnasialquoten beobachtet. Bemerkenswert ist, dass die kleinste Gruppe, die der Katholiken, die zudem (mit Ausnahme des Eichsfelds) über einen historisch längeren Zeitraum in diesem Gebiet quantitativ in der Minderheit war, die höchsten Quoten aufweist. Aus Gründen geringer Fallzahlen können wir aber keine vertiefenden Analysen, z. B. zur selektiven Zuwanderung aus den alten Bundesländern durchführen.

Können wir also das Kapitel Religion und Kirchgang als strukturierende Faktoren für Bildungserfolge ad acta legen? Zumindest für die beiden christlichen Großkonfessionen in Westdeutschland sehen wir dies im Moment so, auch wenn hier nur Gymnasialbeteiligung als Erfolgsindikator untersucht wurde. Der Exkurs zu Ostdeutschland könnte aber auch ein Hinweis darauf sein, dass ein weiteres Fortschreiten der Säkularisierung durch Kirchenaustritte und Abnahme an (Kinds-)Taufen dazu führt, dass Mitglieder der christlichen Großkirchen in Zukunft eine selektive Population darstellen werden, deren Kinder höheren Bildungserfolg aufweisen. Im Fall der Muslime sollten die Ergebnisse mit deutlich größeren Stichproben repliziert werden. Hier hatten wir nur 259 Beobachtungen. Es ist auch darauf hinzuweisen, dass erst nach Kontrolle der sozialstrukturellen Merkmale der Familie der große Abstand zu den katholischen und evangelischen Kindern schwindet. Falls Eltern auf dem Arbeitsmarkt aufgrund ihrer Zugehörigkeit zum Islam Nachteile haben, dann würde die „Bright Boundary“, die nach Alba zentrale Ursache ist, sich über diesen Weg auf die Bildungsbeteiligung des Kindes auswirken.

Für alle betrachten Gruppen gilt, dass die Beteiligung an universitärer Ausbildung, curriculare Leistungen und kognitive Kompetenzen, wie sie in Schulleistungsstudien gemessen werden, unbeobachtet bleiben. Zudem stand uns als Indikator für Religiosität lediglich der Kirchgang zur Verfügung. Kirchgang kann aber auch durch nicht religionsbezogene Aspekte motiviert sein. Indikatoren zu anderen religiösen Prakti- 
ken (z. B. Gebete zuhause) und zu religiösen Überzeugungen liegen in den verwendeten Daten nicht vor.

Zudem beruhen unsere Modelle mit festen Effekten für Kreise auf der Annahme, dass die unbeobachteten Merkmale in den Jahren 1997 bis 2011 konstant waren und dass sie für alle Teilnehmer gleichermaßen wirken. Gerade in Landkreisen mag es auch weiterhin Zentrum-Peripherie-Unterschiede geben, wenn einige Jugendliche vom Schulstandort weit entfernt wohnen und lange Anfahrtswege haben. Dies sollte aber nur dann relevant sein, wenn sich Katholiken, Protestanten und Muslime auch innerhalb von Landkreisen ungleich verteilen. Ein weiteres Problem könnte darin liegen, dass sich Merkmale von Kreisen auf einzelne Gruppen unterschiedlich auswirken. Schulen in konfessioneller Trägerschaft haben häufig Schüler mit eben dieser Konfession. In unserer Studie haben wir als Erfolgsindikator den Besuch eines Gymnasiums ausgewählt. Im unteren und mittleren Sekundarbereich haben Ersatzschulen in konfessioneller Trägerschaft keinen Schwerpunkt auf einer einzigen Schulart; es werden deshalb auch nicht nur Gymnasien in konfessioneller Trägerschaft angeboten. Deshalb bleibt fraglich, ob eine Nicht-Berücksichtigung dieser Schulangebote substanzielle Einflüsse auf die von uns erzielten Ergebnisse hat.

\section{Anhang}

Tab. 7 Gymnasialbesuch in Ostdeutschland - Lineares Wahrscheinlichkeitsmodell. (Quellen: SOEP, v29; eigene Berechnungen)

\begin{tabular}{lcc}
\hline & Modell A1 & Modell A2 \\
\hline Religion der Mutter (Ref.: katholisch, $n=54)$ & $-0,054$ & $(0,078)$ \\
Evangelisch $(n=211)$ & $(0,086)$ & $-0,136^{+}$ \\
& $-0,156^{+}$ & $(0,074)$ \\
Konfessionslos ( $n=651)$ & $(0,081)$ & $0,100^{* *}$ \\
& & $(0,028)$ \\
Häufigkeit Hochkultur (linear) & $0,039^{* *}$ \\
& $(0,008)$ \\
Anzahl Bildungsjahre (Eltern) & $0,004^{* *}$ \\
& $(0,001)$ \\
Höchster ISEI (Eltern) & \\
& & \\
Status Partner der Mutter/Vater & $-0,056$ \\
(Ref:: im HH und Interview) & $(0,043)$ \\
nicht im HH & $-0,044$ \\
im HH, aber kein Interview & $(0,089)$ \\
Geburtsland der Eltern (Ref.: Deutschland) & \\
mind. 1 Elternt. Ausland & 0,025 \\
Junge (Ref.: Mädchen) & $(0,168)$ \\
& $-0,099^{* *}$ \\
\hline
\end{tabular}


Tab. 7 (Fortsetzung)

\begin{tabular}{lcc}
\hline & Modell A1 & Modell A2 \\
\hline Geschwister (Ref.: keine) & & $-0,012$ \\
1 Geschwister. & $(0,035)$ \\
& & $-0,136^{* *}$ \\
mind 2. Geschwister & $(0,039)$ \\
Erhebungsjahr (Ref.: 1997) & \\
2003 & & 0,029 \\
& & $(0,036)$ \\
2007 & & $0,093^{*}$ \\
& & $(0,045)$ \\
2011 & & $0,153^{* *}$ \\
& & $(0,048)$ \\
Konstante & $0,474^{* *}$ \\
Fallzahl (Jugendliche) & $\left(0,019^{* *}\right.$ & 916 \\
$\mathrm{R}^{2}$ & 916 & 0,200 \\
\hline
\end{tabular}

Robuste Standardfehler in Klammern; $\mathrm{n}=$ Fallzahlen

Ref. Referenzkategorie

$+p<0,10 * p<0,05 ; * * p<0,01$;

${ }^{a}$ Mittelwert zentriert

Danksagung: Für wertvolle Impulse zu Vorfassungen möchten wir - in zeitlicher Abfolge - Liliya und Thomas Leopold, den Teilnehmern des SOEP Brown Bag Seminars, insbesondere Alexandra Adveenko, den Gutachtern der Kölner Zeitschrift für Soziologie und Sozialpsychologie und schließlich Ingrid Tucci danken. Ein besonderer Dank gilt auch dem DIW Berlin, das uns mehrfach einen Gastarbeitsplatz gewährte, um mit den Regionaldaten des Sozio-oekonomischen Panels zu rechnen.

\section{Literatur}

Ainsworth-Darnell, James W., und Douglas B. Downey. 1998. Assessing the oppositional culture explanation for racial/ethnic differences in school performance. American Sociological Review 63:536-553.

Alba, Richard. 2005. Bright vs. blurred boundaries: Second-generation assimilation and exclusion in France, Germany, and the United States. Ethnic and Racial Studies 28:20-49.

Becker, Rolf. 2007. Das katholische Arbeitermädchen vom Lande - Ist die Bildungspolitik ein Opfer einer bildungssoziologischen Legende geworden? In Pädagogik und Politik. Historische und aktuelle Perspektiven, Hrsg. Claudia Crotti, Philip Gonon, und Walter Herzog, 177-204. Bern: Haupt.

Becker, Sascha O., und Ludger Wößmann. 2009. Was Weber wrong? A human capital theory of Protestant economic history. The Quaterly Journal of Economics 124:531-596.

Becker, Sascha O., und Ludger Wößmann. 2010. The effect of Protestantism on education before the industrialization: Evidence from 1816 Prussia. Economics Letters 107:224-228.

Blossfeld, Hans-Peter. 1988. Bildungsverläufe im historischen Wandel. Eine Längsschnittanalyse über die Veränderung der Bildungsbeteiligung im Lebenslauf dreier Geburtskohorten. In Bildung, Beruf, Arbeitsmarkt, Hrsg. Hans-Joachim Bodenhöfer, 259-302. Berlin: Duncker \& Humblot.

Connor, Phillip, und Matthias Koenig. 2013. Bridges and barriers: Religion and immigrant occupational attainment across integration contexts. International Migration Review 47:3-38.

Duncan, Greg J., und Stephen W. Raudenbush. 2001. Getting context right in quantitative studies of child development. In The well-being of children and families. Research and data needs, Hrsg. Arland Thornton, 357-383. Ann Arbor: Univ. of Michigan Press. 
Durkheim, Emile. 1983. Der Selbstmord.1897. Frankfurt a. M.: Suhrkamp.

Erlinghagen, Karl. 1965. Katholisches Bildungsdefizit in Deutschland. Freiburg: Herder.

Fitzi, Gregor. 2008. Max Weber. Frankfurt a. M.: Campus.

Foner, Nancy, und Richard Alba. 2008. Immigrant religion in the U.S. and Western Europe: Bridge or barrier to inclusion? International Migration Review 42:360-392.

Fordham, Signithia, und John U. Ogbu. 1987. Black students' school success: Coping with the „burden of ,acting white“". Urban Review 18:176-206.

FORSA - Gesellschaft für Sozialforschung und statistische Analyse mbH. 2008. Forsa-Bus 2007. GESIS Datenarchiv, Köln. ZA4552 Datenfile Version 1.0.0, doi:10.4232/1.4552.

Ganzeboom, Harry B. G., und Donald J. Treiman. 2003. Three internationally standardised measures for comparative research on occupational status. In Advances in cross-national comparison. A European working book for demographic and socio-economic variables, Hrsg. Jürgen H. P. Hoffmeyer-Zlotnik und Christof Wolf, 159-193. New York: Kluwer Academic/Plenum Publishers.

Helbig, Marcel, und Thorsten Schneider. 2014. Auf der Suche nach dem katholischen Arbeitermädchen vom Lande - Religion und Bildungserfolg im regionalen und historischen und internationalen Vergleich (unter Mitarbeit von Juila Dohrmann und Andrea Palasciano). Wiesbaden: Springer VS.

Hoffmann-Lange, Ursula. 1984. Katholiken und Protestanten in der deutschen Führungsschicht. Ausmaß, Ursachen und Bedeutung ungleicher Vertretung von Katholiken und Protestanten in den Eliten der Bundesrepublik. In Konfession - eine Nebensache? Politische, soziale und kulturelle Ausprägungen religiöser Unterschiede in Deutschland, Hrsg. Gerhard Schmidtchen und Hans-Georg Wehling, 75-93. Stuttgart: Kohlhammer.

Jungbauer-Gans, Monika, Henning Lohmann und C. Katharina Spieß. 2012. Bildungsungleichheiten und Privatschulen in Deutschland. In Kölner Zeitschrift für Soziologie und Sozialpsychologie, Sonderheft 52/2012 „Soziologische Bildungsforschung “, Hrsg. Rolf Becker und Heike Solga, 64-85. Wiesbaden: VS Verlag für Sozialwissenschaften.

Kiefer, Amy K., und Denise Sekaquaptewa. 2007. Implicit stereotypes, gender identification, and mathrelated outcomes: A prospective study of female college students. Psychological Science 18:13-18.

Kühnel, Steffen, und Anja Mays. 2010. Lässt sich in der Langzeitbeobachtung ehemaliger Gymnasiasten eine protestantische Ethik finden? Empirische Spurensuche zu Max Weber in Heiner Meulemanns Gymnasiastenpanel. In Komparative empirische Sozialforschung. Anwendungsfelder und aktuelle Methoden in Best Practice-Studien, Hrsg. Tilo Beckers, Klaus W. Birkelbach, Jörg Hagenah und Ulrich Rosar, 93-111. Wiesbaden: VS Verlag für Sozialwissenschaften.

Luther, Martin. 1888. An den christlichen Adel deutscher Nation von des christlichen Standes Besserung. D. Martin Luthers Werke: Kritische Gesamtausgabe. Bd. 6. 1520. Weimar: Hermann Böhlaus Nachfolger.

Mokrosch, Reinhold. 2006. Erziehung und Bildung aus lutherischer Perspektive. In Profilierte Bildung. Der Beitrag der christlichen Kirchen zu den Bildungsaufgaben der Gegenwart, Hrsg. Reinhard Schmidt-Rost, Norbert Dennerlein und Udo Hahn, 9-27. Hannover: Lutherisches Kirchenamt.

Müller, Walter. 1998. Erwartete und unerwartete Folgen der Bildungsexpansion. In Die Diagnosefähigkeit der Soziologie, Hrsg. Jürgen Friedrichs, Rainer M. Lepsius und Karl Ulrich, 81-112. Opladen: Westdeutscher Verlag.

Nellessen-Schumacher, Traute. 1978. Sozialprofil der deutschen Katholiken. Eine konfessionsstatistische Analyse. Mainz: Matthias-Grünewald-Verlag.

Ogbu, John U. 1990. Minority education in comparative perspective. The Journal of Negro Education 59:45-57.

Patacchini, Elonora, und Yves Zenou. 2007. Intergenerational education transmission: neighborhood quality and/or parents' involvement? IZA Discussion Paper No. 2608.

Peisert, Hansgert. 1967. Soziale Lage und Bildungschancen in Deutschland. München: Piper.

Picht, Georg. 1964. Die deutsche Bildungskatastrophe. Freiburg: dtv.

Pickel, Gert. 2013. Die Situation der Religion in Deutschland - Rückkehr des Religiösen oder voranschreitende Säkularisierung? In Religion und Politik im vereinigten Deutschland: Was bleibt von der Rückkehr des Religiösen?, Hrsg. Gert Pickel und Oliver Hidalgo, 65-101. Wiesbaden: VS Verlag für Sozialwissenschaften.

Relikowski, Ilona, Erbil Yilmaz und Hans-Peter Blossfeld. 2012. Wie lassen sich die hohen Bildungsaspirationen von Migranten erklären? Eine Mixed-Methods-Studie zur Rolle von strukturellen Aufstiegschancen und individueller Bildungserfahrung. In Kölner Zeitschrift für Soziologie und Sozialpsychologie, Sonderheft 52/2012 ,Soziologische Bildungsforschung“, Hrsg. Rolf Becker und Heike Solga, 111-136. Wiesbaden: Springer VS. 
Schaltegger, Christoph A, und Benno Torgler. 2010. Work ethic, Protestantism, and human capital. Economics Letters 107:99-101.

Schmidtchen, Gerhard. 1979. Protestanten und Katholiken. Soziologische Analyse konfessioneller Kultur. Bern: Francke.

Schreiner, Martin. 1996. Im Spielraum der Freiheit. Evangelische Schulen als Lernorte Christlicher Weltverantwortung. Göttingen: Vandenhoeck \& Ruprecht.

Solga, Heike, und Rolf Becker. 2012. Soziologische Bildungsforschung - eine kritische Bestandsaufnahme. In Kölner Zeitschrift für Soziologie und Sozialpsychologie, Sonderheft 52/2012 „Soziologische Bildungsforschung “, Hrsg. Rolf Becker und Heike Solga, 7-43. Wiesbaden: Springer VS.

Sozio-oekonomisches Panel (SOEP). 2012. Daten der Jahre 1984-2012, Version 29, SOEP, 2012, doi:10.5684/soep.v29.

Steele, Claude M. 1997. A threat in the air: How stereotypes shape intellectual identity and performance. American Psychologist 52:613-629.

Wagner, Gert G., Jan Göbel, Peter Krause, Rainer Pischner und Ingo Sieber. 2008. Das Sozio-oekonomische Panel (SOEP): Multidisziplinäres Haushaltspanel und Kohortenstudie für Deutschland - Eine Einführung (für neue Datennutzer) mit einem Ausblick (für erfahrene Anwender). Wirtschafts- und Sozialstatistisches Archiv 2:301-328.

Weber, Max. 2006. Religion und Gesellschaft. Gesammelte Aufsätze zur Religionssoziologie. 1904/5, 1920. Frankfurt a. M.: Zweitausendeins.

Wohlrab-Sahr, Monika. 2009. Das stabile Drittel: Religionslosigkeit in Deutschland. In Woran glaubt die Welt? Analysen und Kommentare zum Religionsmonitor 2008, Hrsg. Bertelsmann Stiftung, 151-168. Gütersloh: Verlag Bertelsmann Stiftung.

Woodcock, Anna, Paul R. Hernandez, Mica Estrada und Wesley P. Schultz. 2012. The consequences of chronic stereotype threat: Domain disidentification and abandonment. Journal of Personality and Social Psychology 103:635-646.

Wooldridge, Jeffrey M. 2000. Introductory econometrics. A modern approach. Cincinnati: South-Western College Publishing.

Zacharski, Richard. 1992. Bildung und Religionszugehörigkeit. Auswertung aus der Volkszählung 1987. Beiträge zur Hochschulforschung 4:395-419.

Thorsten Schneider, 1973, Prof. Dr. phil, Professur für Soziologie mit Schwerpunkt Vergleich moderner Gegenwartsgesellschaften, Institut für Soziologie, Universität Leipzig, Forschungsgebiete: Empirische Bildungsforschung, vergleichende Sozialstrukturanalyse, Generationenbeziehungen, Methoden der Längsschnittanalyse. Veröffentlichungen: Auf der Suche nach dem katholischen Arbeitermädchen vom Lande - Religion und Bildungserfolg im regionalen und historischen und internationalen Vergleich. Wiesbaden 2014 (mit M. Helbig); The National Educational Panel Study: Need, Main Features, and Research Potential. Zeitschrift für Erziehungswissenschaft, Sonderheft 14. Wiesbaden 2011 (mit H.-P. Blossfeld u. J. von Maurice).

Julia Dohrmann, 1986, Dipl.-Soz., Doktorandin am Deutschen Institut für Internationale Pädagogische Forschung (DIPF), Frankfurt a. M.. Forschungsgebiete: Schulqualität, Bildungssoziologie. 